\title{
Some characterizations of parallel hyperplanes in multi-layered heat conductors *
}

\author{
Shigeru Sakaguchi ${ }^{\dagger}$ \\ Dedicated to Masaru Ikehata on the occasion of his 60th birthday
}

\begin{abstract}
We consider the Cauchy problem for the heat diffusion equation in the whole space consisting of three layers with different constant conductivities, where initially the upper and middle layers have temperature 0 and the lower layer has temperature 1. Under some appropriate conditions, it is shown that, if either the interface between the lower layer and the middle layer is a stationary isothermic surface or there is a stationary isothermic surface in the middle layer near the lower layer, then the two interfaces must be parallel hyperplanes. Similar propositions hold true, either if a stationary isothermic surface is replaced by a surface with the constant flow property or if the Cauchy problem is replaced by an appropriate initial-boundary value problem.
\end{abstract}

\section{Résumé}

Nous considérons le problème de Cauchy pour l'équation de diffusion de la chaleur dans tout l'espace composé de trois couches avec différentes conductivités constantes, où initialement les couches supérieure et moyenne ont la température 0 et la couche inférieure a la température 1. Dans certaines conditions appropriées, il est montré que, si l'interface entre la couche inférieure et la couche intermédiaire est une surface isotherme stationnaire ou s'il existe une surface isothermique stationnaire dans la couche intermédiaire près de la couche inférieure, alors les deux interfaces doivent être des hyperplans parallèles. Des propositions similaires sont vraies, soit si une surface isotherme stationnaire est remplacée par une surface avec la propriété d'écoulement constant ou si le problème de Cauchy est remplacé par un problème de valeur de limite initiale approprié.

*This research was partially supported by the Grants-in-Aid for Scientific Research (B) ( 18 H01126 and $\sharp 17 \mathrm{H} 02847$ ) of Japan Society for the Promotion of Science.

${ }^{\dagger}$ Research Center for Pure and Applied Mathematics, Graduate School of Information Sciences, Tohoku University, Sendai, 980-8579, Japan. (sigersak@tohoku.ac.jp). 
Key words. heat diffusion equation, multi-layered heat conductors, stationary isothermic surface, constant flow property

AMS subject classifications. Primary 35K05 ; Secondary 35K10, 35B06, 35B40, 35K15, 35K20, 35J05, 35J25

\section{Introduction}

For $x \in \mathbb{R}^{N}$ with $N \geq 2$, set $x=\left(x_{1}, \ldots, x_{N-1}, x_{N}\right)=\left(y, x_{N}\right)$ for $y \in \mathbb{R}^{N-1}$. Let $f, h \in C^{2}\left(\mathbb{R}^{N-1}\right)$ satisfy

$$
f(y)<h(y) \text { for every } y \in \mathbb{R}^{N-1} \text {. }
$$

Define two domains $D, \Omega$ in $\mathbb{R}^{N}$ by

$$
D=\left\{x \in \mathbb{R}^{N}: x_{N}>h(y)\right\}, \quad \Omega=\left\{x \in \mathbb{R}^{N}: x_{N}>f(y)\right\}
$$

respectively. Denote by $\sigma=\sigma(x)\left(x \in \mathbb{R}^{N}\right)$ the conductivity distribution of the whole medium given by

$$
\sigma= \begin{cases}\sigma_{c} & \text { in } D, \\ \sigma_{s} & \text { in } \Omega \backslash D, \\ \sigma_{m} & \text { in } \mathbb{R}^{N} \backslash \Omega,\end{cases}
$$

where $\sigma_{c}, \sigma_{s}, \sigma_{m}$ are positive constants with $\sigma_{c} \neq \sigma_{s}$. This kind of three-phase electrical conductor has been dealt with in [9] in the study of neutrally coated inclusions.

Let $u=u(x, t)$ be the unique bounded solution of either the Cauchy problem for the heat diffusion equation:

$$
u_{t}=\operatorname{div}(\sigma \nabla u) \quad \text { in } \mathbb{R}^{N} \times(0,+\infty) \text { and } u=\mathcal{X}_{\Omega^{c}} \text { on } \mathbb{R}^{N} \times\{0\}
$$

where $\mathcal{X}_{\Omega^{c}}$ denotes the characteristic function of the set $\Omega^{c}=\mathbb{R}^{N} \backslash \Omega$, or the initialboundary value problem for the heat diffusion equation:

$$
\begin{array}{ll}
u_{t}=\operatorname{div}(\sigma \nabla u) & \text { in } \Omega \times(0,+\infty), \\
u=1 & \text { on } \partial \Omega \times(0,+\infty), \\
u=0 & \text { on } \Omega \times\{0\} .
\end{array}
$$

Let $g \in C^{0}\left(\mathbb{R}^{N-1}\right)$ satisfy

$$
f(y)<g(y)<h(y) \text { for every } y \in \mathbb{R}^{N-1} \text {. }
$$


Consider a domain $G$ in $\mathbb{R}^{N}$ defined by

$$
G=\left\{x \in \mathbb{R}^{N}: x_{N}>g(y)\right\}
$$

Suppose that

$$
\operatorname{dist}(x, \partial \Omega) \leq \operatorname{dist}(x, \bar{D}) \text { for every } x \in \partial G .
$$

This assumption is technical and corresponds to those in [15, (5)], [16, (1.5)] and [4, (1.6)], and it enables us to utilize the balance laws [10, Theorem 2.1 and Corollary 2.2].

Let us first state two theorems concerning stationary isothermic surfaces.

Theorem 1.1 Either let $N \leq 8$ or let $\nabla f$ be bounded in $\mathbb{R}^{N-1}$ with $N \geq 2$. Suppose that $\partial \Omega$ is uniformly of class $C^{6}$ and the function $h-f$ has a minimum value in $\mathbb{R}^{N-1}$ and moreover, either $h-f$ has a maximum value in $\mathbb{R}^{N-1}$ or $h-f$ is unbounded in $\mathbb{R}^{N-1}$. Let $u$ be the solution of problem (1.3). If there exists a function $a:(0,+\infty) \rightarrow(0,+\infty)$ satisfying

$$
u(x, t)=a(t) \text { for every }(x, t) \in \partial \Omega \times(0,+\infty),
$$

then $\partial \Omega$ and $\partial D$ must be parallel hyperplanes.

Theorem 1.2 Either let $N \leq 3$ or let $\{|f(y)-f(\hat{y})|:|y-\hat{y}| \leq 1\}$ be bounded. Suppose that the function $h-f$ has a minimum value in $\mathbb{R}^{N-1}$ and either $h-f$ has a maximum value in $\mathbb{R}^{N-1}$ or $h-f$ is unbounded in $\mathbb{R}^{N-1}$. Let $u$ be the solution of problem (1.3) or problem (1.4) -(1.6). Under the assumption (1.8), if there exists a function $a:(0,+\infty) \rightarrow(0,+\infty)$ satisfying

$$
u(x, t)=a(t) \text { for every }(x, t) \in \partial G \times(0,+\infty),
$$

then $\partial \Omega$ and $\partial D$ must be parallel hyperplanes.

In Theorems 1.1 and 1.2, the conditions (1.9) and (1.10) mean that each of $\partial \Omega$ and $\partial G$ is a stationary isothermic surface. Thus each of Theorems 1.1 and 1.2 characterizes parallel hyperplanes as the interfaces in such a way that there exists a stationary isothermic surface in the multi-layered heat conductors. The assumptions on the function $h-f$ are technical, and in particular the existence of its maximum value or its minimum value enables us to utilize Hopf's boundary point lemma.

Next two theorems replace a stationary isothermic surface by a surface with the constant flow property which was dealt with in [4].

Theorem 1.3 Either let $N \leq 8$ or let $\nabla f$ be bounded in $\mathbb{R}^{N-1}$ with $N \geq 2$. Suppose that $\partial \Omega$ is uniformly of class $C^{6}$ and the function $h-f$ has a minimum value in $\mathbb{R}^{N-1}$ and 
moreover, either $h-f$ has a maximum value in $\mathbb{R}^{N-1}$ or $h-f$ is unbounded in $\mathbb{R}^{N-1}$. Let $u$ be the solution of problem (1.4) -(1.6). If there exists a function $b:(0,+\infty) \rightarrow \mathbb{R}$ satisfying

$$
\sigma_{s} \frac{\partial u}{\partial \nu}(x, t)=b(t) \text { for every }(x, t) \in \partial \Omega \times(0,+\infty),
$$

then $\partial \Omega$ and $\partial D$ must be parallel hyperplanes, where $\nu$ denotes the outward unit normal vector to $\partial \Omega$.

Theorem 1.4 Either let $N \leq 3$ or let $\{|f(y)-f(\hat{y})|:|y-\hat{y}| \leq 1\}$ be bounded. Suppose that the function $h-f$ has a minimum value in $\mathbb{R}^{N-1}$ and either $h-f$ has a maximum value in $\mathbb{R}^{N-1}$ or $h-f$ is unbounded in $\mathbb{R}^{N-1}$, and moreover $g \in C^{1}\left(\mathbb{R}^{N-1}\right)$. Let $u$ be the solution of problem (1.3) or problem (1.4)-(1.6). Under the assumption (1.8), if there exists a function $b:(0,+\infty) \rightarrow \mathbb{R}$ satisfying

$$
\sigma_{s} \frac{\partial u}{\partial \nu}(x, t)=b(t) \text { for every }(x, t) \in \partial G \times(0,+\infty),
$$

then $\partial \Omega$ and $\partial D$ must be parallel hyperplanes, where $\nu$ denotes the outward unit normal vector to $\partial G$.

In Theorem 1.3 the condition (1.11), together with the boundary condition (1.5), is overdetermined and it implies that the heat flow is parallel to the normal vector to $\partial \Omega$ and the amount of the flow is constant on $\partial \Omega$ for each time. Such a condition was given by [1, 6] for parabolic problems, which generalizes the overdetermined condition of Serrin [17] for elliptic problems. Recently such a boundary $\partial \Omega$ was called a surface with the constant flow property in the context of the heat flow in smooth Riemannian manifolds by [13]. The condition (1.12), which was introduced by [4], is an overdetermination different from Serrin-type, and we still called it the constant flow property in [4]. Similar characterizations of concentric balls in multi-phase heat conductors were obtained in the previous papers [15, 16, 4], and in the present paper we deal with hyperplanes, which are not compact and need additional cares. The proofs of all the theorems consist of two steps. In the first step we show that $\partial \Omega$ must be a hyperplane, and the second step is devoted to proving that $\partial D$ is a hyperplane parallel to $\partial \Omega$. We have two strategies in the first step; one applies to Theorems 1.2 and 1.4 and the other does to Theorems 1.1 and 1.3. On the other hand, the second step follows from one strategy common to all the theorems, which depends on a result concerning an elliptic overdetermined problem (see Theorem 5.1 in section (5).

The following sections are organized as follows. In section 2, we recall one lemma and three propositions from [4, 15], where we need to modify the two propositions in order to 
deal with the case where $\partial \Omega$ is unbounded. Indeed, we show that our case is reduced to the case where $\partial \Omega$ is bounded and of class $C^{2}$ with the aid of the maximum principle and the Gaussian bounds for the fundamental solution of $u_{t}=\operatorname{div}(\sigma \nabla u)$ due to Aronson [2, Theorem 1, p. 891](see also [5, p. 328]). Section 3 is devoted to the proofs of Theorems 1.2 and 1.4, the balance laws (Proposition 2.4) and the asymptotic formula of the heat content of balls touching at a point on $\partial \Omega$ (Proposition 2.2) play a key role to show that $\partial \Omega$ must be a sort of Weingarten surface, and hence some results of [14] implies that $\partial \Omega$ is a hyperplane. Finally, by using Theorem 5.1 given in section 5 , which concerns an elliptic overdetermined problem, we complete the proofs through the Laplace transform. Section 4 is devoted to the proofs of Theorems 1.3 and 1.1. Under the assumption that $\partial \Omega$ is uniformly of class $C^{6}$, the same arguments with the precise barriers as in the proofs of [4, Theorems 1.4 and 1.5 in section 5] work and we conclude that the mean curvature of $\partial \Omega$ must be constant even if $\partial \Omega$ is unbounded. Hence both the Bernstein theorem and Moser's theorem for the minimal surface equation imply that $\partial \Omega$ is a hyperplane under appropriate assumptions. Finally, Theorem 5.1 completes the proofs through the Laplace transform. In section 5, we give a proof of Theorem 5.1, where Hopf's boundary point lemma and the transmission condition on $\partial D$, together with three comparison principles and one maximum principle for elliptic equations with discontinuous conductivities given in section 6, play a key role. Roughly, Theorem 5.1 states that if $\partial \Omega$ is a hyperplane then $\partial D$ must be a hyperplane parallel to $\partial \Omega$. The last section 6 is devoted to the proofs of three comparison principles and one maximum principle for elliptic equations with discontinuous conductivities.

\section{Preliminaries}

Let us introduce the distance function $\delta=\delta(x)$ of $x \in \mathbb{R}^{N}$ to $\partial \Omega$ by

$$
\delta(x)=\operatorname{dist}(x, \partial \Omega) \text { for } x \in \mathbb{R}^{N}
$$

We quote a lemma concerning the solutions of problem (1.3) and problem (1.4)-(1.6) from [4. Lemma 4.1], which simply comes from the maximum principle and the Gaussian bounds for the fundamental solution of $u_{t}=\operatorname{div}(\sigma \nabla u)$ due to Aronson [2, Theorem 1, p. 891] (see also [5, p. 328]). Although [4, Lemma 4.1] concerns the case where $\Omega$ is bounded, exactly the same proof is applicable even if $\Omega$ is unbounded. For $\tau>0$, we set

$$
\Omega_{\tau}=\{x \in \Omega: \delta(x) \geq \tau\} \text { and } \Omega_{\tau}^{c}=\left\{x \in \mathbb{R}^{N} \backslash \Omega: \delta(x) \geq \tau\right\} .
$$


Lemma 2.1 Let $u$ be the solution of either problem (1.3) or problem (1.4)-(1.6) with a general conductivity $\sigma=\sigma(x)\left(x \in \mathbb{R}^{N}\right)$ satisfying

$$
0<\mu \leq \sigma(x) \leq M \text { for every } x \in \mathbb{R}^{N}
$$

where $\mu, M$ are positive constants. Then the following propositions hold true:

(1) The solution u satisfies

$$
0<u<1 \text { in } \mathbb{R}^{N} \times(0,+\infty) \text { or in } \Omega \times(0,+\infty), \text { respectively. }
$$

(2) For every $\tau>0$, there exist two positive constants $B$ and $b$ such that

$$
0<u(x, t)<B e^{-\frac{b}{t}} \quad \text { for every }(x, t) \in \Omega_{\tau} \times(0,+\infty)
$$

and, moreover, if $u$ is the solution of (1.3), then

$$
0<1-u(x, t)<B e^{-\frac{b}{t}} \quad \text { for every }(x, t) \in \Omega_{\tau}^{c} \times(0,+\infty) .
$$

(3) The solution $u$ of (1.3) is such that

$$
\lim _{x \notin \Omega, \delta(x) \rightarrow \infty}(1-u(x, t))=0 \quad \text { for every } t \in(0,+\infty) .
$$

In [4, Theorems 1.3 and 1.2], a proposition ([15, Proposition 2.2, pp. 171-172]) plays a key role, where the boundary of the domain is compact. Here, we deal with the case where $\partial \Omega$ is unbounded, and therefore we need to modify the proposition. Denote by $B_{r}(x)$ an open ball in $\mathbb{R}^{N}$ with a radius $r>0$ and centered at a point $x \in \mathbb{R}^{N}$. The modified one is the following:

Proposition 2.2 Let $\Omega$ be a possibly unbounded domain in $\mathbb{R}^{N}$, and let $x_{0} \in \Omega$ and $z_{0} \in \partial \Omega$. Assume that $B_{r}\left(x_{0}\right) \subset \Omega, \overline{B_{r}\left(x_{0}\right)} \cap \partial \Omega=\left\{z_{0}\right\}$ and there exists $\varepsilon>0$ such that $\partial \Omega \cap B_{\varepsilon}\left(z_{0}\right)$ is of class $C^{2}$ and $\partial \Omega$ divides $B_{\varepsilon}\left(z_{0}\right)$ into two connected components. Let $\sigma=\sigma(x)\left(x \in \mathbb{R}^{N}\right)$ be a general conductivity satisfying

$$
0<\mu \leq \sigma(x) \leq M \quad \text { for every } x \in \mathbb{R}^{N}, \text { and } \sigma(x)= \begin{cases}\sigma_{s} & \text { if } x \in B_{\varepsilon}\left(z_{0}\right) \cap \Omega, \\ \sigma_{m} & \text { if } x \in B_{\varepsilon}\left(z_{0}\right) \backslash \Omega,\end{cases}
$$

where $\mu, M, \sigma_{s}$, and $\sigma_{m}$ are positive constants. Let $u$ be the bounded solution of either problem (1.3) or problem (1.4)-(1.6) for this general conductivity $\sigma$. Then we have:

$$
\lim _{t \rightarrow+0} t^{-\frac{N+1}{4}} \int_{B_{r}\left(x_{0}\right)} u(x, t) d x=C(N, \sigma)\left\{\prod_{j=1}^{N-1}\left(\frac{1}{r}-\kappa_{j}\left(z_{0}\right)\right)\right\}^{-\frac{1}{2}} .
$$


Here, $\kappa_{1}\left(z_{0}\right), \ldots, \kappa_{N-1}\left(z_{0}\right)$ denote the principal curvatures of $\partial \Omega$ at $z_{0}$ with respect to the inward normal direction to $\partial \Omega$ and $C(N, \sigma)$ is a positive constant given by

$$
C(N, \sigma)=\left\{\begin{array}{cl}
2 \sigma_{s}^{\frac{N+1}{4}} c(N) & \text { for problem (1.4) }-(1.6), \\
\frac{2 \sqrt{\sigma_{m}}}{\sqrt{\sigma_{s}}+\sqrt{\sigma_{m}}} \sigma_{s}^{\frac{N+1}{4}} c(N) & \text { for problem (1.3) }
\end{array}\right.
$$

where $c(N)$ is a positive constant depending only on $N$. (Notice that if $\sigma_{s}=\sigma_{m}$ then $C(N, \sigma)=\sigma_{s}^{\frac{N+1}{4}} c(N)$ for problem (1.3), that is, just half of the constant for problem (1.4) -(1.6).) When $\kappa_{j}\left(z_{0}\right)=1 / r$ for some $j \in\{1, \cdots, N-1\}$, (2.3) holds by setting the right-hand side to $+\infty$ (notice that $\kappa_{j}\left(z_{0}\right) \leq 1 / r$ always holds for all $j$ 's).

Proof. It suffices to show that our case is reduced to the case where $\partial \Omega$ is bounded and of class $C^{2}$. Since $\partial \Omega \cap B_{\varepsilon}\left(z_{0}\right)$ is of class $C^{2}$, we can find a bounded domain $\Omega_{*}$ with $C^{2}$ boundary $\partial \Omega_{*}$ satisfying

$$
B_{r}\left(x_{0}\right) \cup\left(\Omega \cap \overline{B_{\frac{2}{3} \varepsilon}\left(z_{0}\right)}\right) \subset \Omega_{*} \subset \Omega, \overline{B_{\frac{2}{3} \varepsilon}\left(z_{0}\right)} \cap \partial \Omega \subset \partial \Omega_{*} \text { and } \overline{B_{r}\left(x_{0}\right)} \cap \partial \Omega_{*}=\left\{z_{0}\right\} .
$$

Let us first consider problem (1.4)-(1.6). Let $u_{*}=u_{*}(x, t)$ be the bounded solution of problem (1.4)-(1.6) where $\Omega$ and $\sigma$ are replaced with $\Omega_{*}$ and $\sigma_{s}$, respectively. Then, it follows from [15, Proposition 2.2, pp. 171-172] that the formula (2.3) holds true for $u_{*}$. We observe that the difference $v=u-u_{*}$ satisfies

$$
\begin{aligned}
& v_{t}=\sigma_{s} \Delta v \quad \text { in } \quad\left(\Omega \cap B_{\frac{2}{3} \varepsilon}\left(z_{0}\right)\right) \times(0,+\infty), \\
& v=0 \quad \text { on }\left(\partial \Omega \cap \overline{B_{\frac{2}{3} \varepsilon}\left(z_{0}\right)}\right) \times(0,+\infty) \text {, } \\
& |v|<1 \quad \text { on } \Omega_{*} \times(0,+\infty), \\
& v=0 \quad \text { on } \Omega_{*} \times\{0\} .
\end{aligned}
$$

Set

$$
\mathcal{N}=\left\{x \in \mathbb{R}^{N}: \operatorname{dist}\left(x, \Omega_{*} \cap \partial B_{\frac{2}{3} \varepsilon}\left(z_{0}\right)\right)<\frac{1}{100} \varepsilon\right\} .
$$

By comparing $v$ with the solutions of the Cauchy problem for the heat equation with conductivity $\sigma_{s}$ and initial data $\pm 2 \mathcal{X}_{\mathcal{N}}$ for a short time, we see that there exist two positive constants $B$ and $b$ such that

$$
|v(x, t)| \leq B e^{-\frac{b}{t}} \text { for every }(x, t) \in \overline{B_{\frac{1}{2} \varepsilon}\left(z_{0}\right) \cap \Omega} \times(0, \infty) .
$$

By (2) of Lemma 2.1, we may also have

$$
0<u(x, t), u_{*}(x, t) \leq B e^{-\frac{b}{t}} \text { for every }(x, t) \in\left(\overline{B_{r}\left(x_{0}\right)} \backslash B_{\frac{1}{2} \varepsilon}\left(z_{0}\right)\right) \times(0, \infty) .
$$


Then, it follows from (2.8) and (2.9) that $u$ also satisfies (2.3), since we already know that $u_{*}$ satisfies (2.3). Indeed, observing that

$$
t^{-\frac{N+1}{4}} \int_{B_{r}\left(x_{0}\right)} v d x=t^{-\frac{N+1}{4}} \int_{B_{r}\left(x_{0}\right) \backslash B_{\frac{1}{2} \varepsilon}\left(z_{0}\right)} v d x+t^{-\frac{N+1}{4}} \int_{B_{r}\left(x_{0}\right) \cap B_{\frac{1}{2} \varepsilon}\left(z_{0}\right)} v d x
$$

and letting $t \rightarrow \infty$ yield the conclusion.

It remains to consider problem (1.3). Let us define the conductivity $\sigma_{*}=\sigma_{*}(x)(x \in$ $\left.\mathbb{R}^{N}\right)$ by

$$
\sigma_{*}= \begin{cases}\sigma_{s} & \text { in } \Omega_{*} \\ \sigma_{m} & \text { in } \mathbb{R}^{N} \backslash \Omega_{*} .\end{cases}
$$

Let $u_{*}=u_{*}(x, t)$ be the bounded solution of problem (1.3) where $\Omega$ and $\sigma$ are replaced with $\Omega_{*}$ and $\sigma_{*}$, respectively. Then, it follows from [15, Proposition 2.2, pp. 171-172] that the formula (2.3) holds true for $u_{*}$. We observe that the difference $v=u-u_{*}$ satisfies

$$
\begin{array}{ll}
v_{t}=\operatorname{div}\left(\sigma_{*} \nabla v\right) & \text { in } B_{\frac{2}{3} \varepsilon}\left(z_{0}\right) \times(0,+\infty), \\
|v|<1 & \text { in } \mathbb{R}^{N} \times(0,+\infty), \\
v=0 & \text { on }\left(\Omega_{*} \cup \overline{B_{\frac{2}{3} \varepsilon}\left(z_{0}\right)}\right) \times\{0\} .
\end{array}
$$

Then, by the same comparison arguments with the aid of the Gaussian bounds due to Aronson [2, Theorem 1, p. 891] (see also [5, p. 328]), we see that there exist two positive constants $B$ and $b$ satisfying (2.9) and

$$
|v(x, t)| \leq B e^{-\frac{b}{t}} \text { for every }(x, t) \in \overline{B_{\frac{1}{2} \varepsilon}\left(z_{0}\right)} \times(0, \infty),
$$

and hence $u$ also satisfies (2.3)

Since a proposition [4, Proposition E], where the boundary of the domain is compact, also plays a key role in [4, we need to modify the proposition in order to deal with the case where $\partial \Omega$ is unbounded.

Proposition 2.3 Let $\Omega$ be a possibly unbounded domain in $\mathbb{R}^{N}$, and let $z_{0} \in \partial \Omega$. Assume that there exists $\varepsilon>0$ such that $\partial \Omega \cap B_{\varepsilon}\left(z_{0}\right)$ is of class $C^{2}$ and $\partial \Omega$ divides $B_{\varepsilon}\left(z_{0}\right)$ into two connected components. Let $\sigma=\sigma(x)\left(x \in \mathbb{R}^{N}\right)$ be a general conductivity satisfying

$$
0<\mu \leq \sigma(x) \leq M \quad \text { for every } x \in \mathbb{R}^{N}, \text { and } \sigma(x)= \begin{cases}\sigma_{s} & \text { if } x \in B_{\varepsilon}\left(z_{0}\right) \cap \Omega, \\ \sigma_{m} & \text { if } x \in B_{\varepsilon}\left(z_{0}\right) \backslash \Omega,\end{cases}
$$

where $\mu, M, \sigma_{s}$, and $\sigma_{m}$ are positive constants. Let $u$ be the bounded solution of problem (1.3) for this general conductivity $\sigma$. Then, as $t \rightarrow+0$, u converges to the number $\frac{\sqrt{\sigma_{m}}}{\sqrt{\sigma_{s}}+\sqrt{\sigma_{m}}}$ uniformly on $\partial \Omega \cap \overline{B_{\frac{1}{2} \varepsilon}\left(z_{0}\right)}$. 
Proof. It suffices to show that our case is reduced to the case where $\partial \Omega$ is bounded and of class $C^{2}$. As in the proof of Proposition 2.2 for problem (1.3), let $u_{*}=u_{*}(x, t)$ be the bounded solution of problem (1.3) where $\Omega$ and $\sigma$ are replaced with $\Omega_{*}$ and $\sigma_{*}$, respectively. Then $u_{*}$ satisfies the conclusion because of [4, Proposition E]. Therefore, since $v=u-u_{*}$ satisfies (2.14), $u$ also satisfies the conclusion.

We quote another ingredient called a balance law adjusted to our use from [4, Lemma 4.2] and [10, Theorem 2.1]. For convenience, we give a proof with the aid of [10, Theorem $2.1]$.

Proposition $2.4\left([4,[10])\right.$ Let $W$ be a domain in $\mathbb{R}^{N}$ with $N \geq 2$, and let $u=u(x, t)$ satisfy

$$
u_{t}=\sigma_{s} \Delta u \text { in } W \times(0,+\infty) .
$$

Consider two points $p, q \in W$ and two unit vectors $\xi, \eta \in \mathbb{R}^{N}$. Set

$$
r_{*}=\min \{\operatorname{dist}(p, \partial W), \operatorname{dist}(q, \partial W)\}
$$

Then the following three propositions hold true:

(1) $u(p, t)=u(q, t)$ for every $t>0$ if and only if

$$
\int_{B_{r}(p)} u(x, t) d x=\int_{B_{r}(q)} u(x, t) d x \text { for every }(t, r) \in(0,+\infty) \times\left(0, r_{*}\right) .
$$

(2) $\xi \cdot \nabla u(p, t)=\eta \cdot \nabla u(q, t)$ for every $t>0$ if and only if

$$
\xi \cdot \int_{B_{r}(p)} u(x, t)(x-p) d x=\eta \cdot \int_{B_{r}(q)} u(x, t)(x-q) d x \quad \text { for every }(t, r) \in(0,+\infty) \times\left(0, r_{*}\right) .
$$

(3) $\nabla u(p, t)=0$ for every $t>0$ if and only if

$$
\int_{B_{r}(p)} u(x, t)(x-p) d x=0 \text { for every }(t, r) \in(0,+\infty) \times(0, \operatorname{dist}(p, \partial W)) .
$$

Proof. (3) is just [10, Corollary 2.2]. (1) follows from [10, Theorem 2.1]. Indeed, consider the function

$$
v_{1}(x, t)=u(x+p, t)-u(x+q, t) \text { for }(x, t) \in B_{r_{*}}(0) \times(0,+\infty) .
$$

Then $v_{1}$ satisfies the heat equation with conductivity $\sigma_{s}$ and $v_{1}(0, t)=0$ for every $t>0$. Thus [10, Theorem 2.1] gives the conclusion. 
(2) is proved in [4, Lemma 4.2] with the aid of [10, Theorem 2.1]. For (2), by choosing an orthogonal matrix $A$ satisfying $A \xi=\eta$, we consider the function

$$
v_{2}(x, t)=u(x+p, t)-u(A x+q, t) \text { for }(x, t) \in B_{r_{*}}(0) \times(0,+\infty) .
$$

Then the function $\xi \cdot \nabla v_{2}(x, t)$ satisfies the heat equation with conductivity $\sigma_{s}$ and for every $t>0$

$$
\xi \cdot \nabla v_{2}(0, t)=\xi \cdot \nabla u(p, t)-\eta \cdot \nabla u(q, t)=0
$$

Thus, it follows from [10, Theorem 2.1] that

$$
\xi \cdot \int_{B_{r}(0)} \nabla v_{2}(x, t) d x=0 \text { for every }(t, r) \in(0,+\infty) \times\left(0, r_{*}\right),
$$

and hence, by the divergence theorem and again integrating in $r$, we infer that

$$
\xi \cdot \int_{B_{r}(0)} v_{2}(x, t) x d x=0 \text { for every }(t, r) \in(0,+\infty) \times\left(0, r_{*}\right),
$$

which gives (2).

\section{Proofs of Theorems 1.2 and 1.4: the 1st strategy}

Under each of the assumptions of Theorems 1.2 and 1.4, we follow the proofs of [15, Theorems 1.1 and 1.3] and [4, Theorem 1.2], respectively, in order to prove that $\partial \Omega$ is parallel to $\partial G$ and the quantity $\prod_{j=1}^{N-1}\left(1 / R-\kappa_{j}(z)\right)$ is constant for $z \in \partial \Omega$, where $R$ is the distance between $\partial \Omega$ and $\partial G, \kappa_{1}(z), \ldots, \kappa_{N-1}(z)$ denote the principal curvatures of $\partial \Omega$ at a point $z \in \partial \Omega$ with respect to the inward normal direction to $\partial \Omega$, and $\max _{1 \leq j \leq N-1} \kappa_{j}<1 / R$ for every $z \in \partial \Omega$. Once this is proved, we immediately infer that $\partial \Omega$ must be a hyperplane. Indeed, if $N=2$ then $\partial \Omega$ must be a straight line, if $N=3$, by [14, Theorem 4, p. 281], $\partial \Omega$ must be a hyperplane, and if $\{|f(y)-f(\hat{y})|:|y-\hat{y}| \leq 1\}$ is bounded with $N \geq 2$, by [14, Theorem 3 and Remark 3, p. 273], the same conclusion holds true. In the proof of [14. Theorem 4, p. 281], the strong comparison principle for the viscosity solutions of the minimal surface equation plays a key role. Note that [12] gives a simple proof of the strong comparison principle for the prescribed mean curvature equation including the minimal surface equation.

We need to modify [4, Lemma 4.3] in order to deal with the case where $\partial \Omega$ is unbounded and $\partial G$ is of class $C^{1}$ under the assumption (1.12). 
Lemma 3.1 Let $u$ be the solution of either problem (1.3) or problem (1.4)-(1.6). Under each of the assumptions (1.10) and (1.12) of Theorems 1.2 and1.4, the following assertions hold:

(1) there exists a number $R>0$ such that

$$
\delta(x)=R \text { for every } x \in \partial G,
$$

where $\delta(x)$ is the distance function given by (2.1);

(2) $\partial \Omega$ and $\partial G$ are real analytic hypersurfaces;

(3) the mapping $\partial \Omega \ni z \mapsto x(z) \equiv z-R \nu(z) \in \partial G$ is a diffeomorphism where $\nu(z)$ denotes the outward unit normal vector to $\partial \Omega$ at $z \in \partial \Omega$; in particular $\partial \Omega$ and $\partial G$ are parallel hypersurfaces at distance $R$;

(4) the principal curvatures of $\partial \Omega$ satisfy

$$
\max _{1 \leq j \leq N-1} \kappa_{j}(z)<\frac{1}{R} \text { for every } z \in \partial \Omega
$$

(5) there exists a number $c>0$ satisfying

$$
\prod_{j=1}^{N-1}\left(\frac{1}{R}-\kappa_{j}(z)\right)=c \text { for every } z \in \partial \Omega .
$$

Before proving this lemma, we prepare a purely geometric lemma for the proof of Theorem 1.4.

Lemma 3.2 Suppose that $g \in C^{1}\left(\mathbb{R}^{N-1}\right)$ in the definition (1.7) of $G$. Set

$$
R=\inf \{\delta(x): x \in \partial G\}(\geq 0)
$$

where $\delta(x)$ is the distance function given by (2.1). Then, for every $\varepsilon>0$, there exists a point $p \in \partial G$ such that

$$
\begin{aligned}
& \delta(p)<R+\varepsilon ; \\
& \text { there exists a point } z \in \partial \Omega \text { with } B_{\delta(p)}(p) \cap \partial \Omega=\{z\} ; \\
& (z-p) \cdot \nu(p) \neq 0 \text { and } \max _{1 \leq j \leq N-1} \kappa_{j}(z)<\frac{1}{\delta(p)}
\end{aligned}
$$

where $\nu(p)$ denotes the outward unit normal vector to $\partial G$ at $p \in \partial G$. 
Proof. Let $\varepsilon>0$. Set

$$
G_{\varepsilon}=\left\{x \in \mathbb{R}^{N}: x_{N}>g(y)+\frac{\varepsilon}{2}\right\} .
$$

Since $\inf \left\{\delta(x): x \in \partial G_{\varepsilon}\right\} \leq R+\frac{\varepsilon}{2}$, there exists a point $q \in \partial G_{\varepsilon}$ with $\delta(q)<R+\varepsilon$. Then there exists $z \in \partial \Omega$ with $\delta(q)=|q-z|$. By the intermediate value theorem there exists a point $p \in \partial G \cap \overline{q z}$ such that

$$
|p-z|<|q-z|<R+\varepsilon
$$

where $\overline{q z}$ denotes the line segment connecting $q$ and $z$. Therefore we infer that

$$
B_{\delta(p)}(p) \cap \partial \Omega=\{z\} \text { and } \max _{1 \leq j \leq N-1} \kappa_{j}(z) \leq \frac{1}{\delta(q)}<\frac{1}{\delta(p)} .
$$

Hence, by the inverse mapping theorem and (3.5), there exists an infinite solid cylinder $U$, whose axis is the line containing $\overline{q z}$, such that

$$
\delta \in C^{2}(\overline{U \cap(\Omega \backslash G)}) \text { and } \nabla \delta(p)=\frac{p-z}{|p-z|} .
$$

If $\nabla \delta(p) \cdot \nu(p) \neq 0$, then the conclusion follows from (3.5). Thus, let us consider the case where $\nabla \delta(x) \cdot \nu(x)=0$ for all $x \in U \cap(\Omega \backslash G) \cap \partial G$. Let $x=x(s)(s \in \mathbb{R})$ the curve determined by the Cauchy problem:

$$
\frac{d}{d s} x(s)=-\nabla \delta(x(s)) \text { and } x(0)=p .
$$

Then, as long as $x(s)$ exists, $x(s) \in \partial G$ and moreover, since $\nabla \delta(x)=\frac{p-z}{|p-z|}$ for every $x \in \overline{p z}$, we have from the uniqueness of the solution of the Cauchy problem (3.6)

$$
x(s)=p-s \frac{p-z}{|p-z|} .
$$

These contradict the fact that $\delta(x(s)) \geq R$ and $\delta(x(s))=-s+\delta(p)$. Thus there exists a point $x \in U \cap(\Omega \backslash G) \cap \partial G$ with $\nabla \delta(x) \cdot \nu(x) \neq 0$. This point $x \in \partial G$ replaces $p$.

Proof of Lemma 3.1. First, it follows from the assumption (1.8) that

$$
B_{r}(x) \subset \Omega \backslash \bar{D} \text { for every } x \in \partial G \text { with } 0<r \leq \delta(x) .
$$

Therefore, since $\sigma=\sigma_{s}$ in $\Omega \backslash \bar{D}$, we can use Lemma 2.4.

Let us first deal with Theorem 1.2. Then, with the aid of Lemma 2.4, Lemma 2.1 and Proposition 2.2, under the assumption (1.10) of Theorem 1.2 the same proof as in 15, Lemma 2.4, pp. 176-179] is applicable in showing all the assertions (1)-(5) of this lemma even if $\partial \Omega$ is not compact. Roughly, suppose that $\delta(p)<\delta(q)$ for some points $p, q \in \partial G$. 
Then, (1.10) gives (1) of Proposition 2.4. In particular, we choose $r=\delta(p)$. On the other hand, combining (2) of Lemma 2.1 and Proposition 2.2 yields a contradiction to (1) of Proposition 2.4 with $r=\delta(p)$. Thus assertion (1) holds under the assumption (1.10). Once we have (1) under the assumption (1.10) of Theorem 1.2, the others (2)-(5) follow easily. In particular, the analyticity of $\partial G$ follows from the analyticity of the solution $u=u(x, t)$ in $x$, if one shows that for every $x \in \partial G$ there exists a time $t>0$ satisfying $\nabla u(x, t) \neq 0$ with the aid of (1.10), (3) of Lemma 2.4, (2) of Lemma 2.1 and Proposition 2.2. $\partial \Omega$ is also real analytic by (3).

Let us proceed to Theorem 1.4. Since [4, Lemma 4.3] concerns the case where $\partial \Omega$ is compact and $\partial G$ is of class $C^{2}$, we need to modify its proof in order to deal with the case where $\partial \Omega$ is not compact and $\partial G$ is of class $C^{1}$. Let us consider assertion (1) under the assumption (1.12) of Theorem 1.4. Let $\varepsilon>0$. Then it follows from Lemma 3.2 that there exists a point $p \in \partial G$ satisfying (3.2)-(3.4). Hence it follows from Proposition 2.2 and (2) of Lemma 2.1 that

$$
\lim _{t \rightarrow+0} t^{-\frac{N+1}{4}} \nu(p) \cdot \int_{B_{\delta(p)}(p)} u(x, t)(x-p) d x=C(N, \sigma) \nu(p) \cdot(z-p)\left\{\prod_{j=1}^{N-1}\left(\frac{1}{\delta(p)}-\kappa_{j}(z)\right)\right\}^{-\frac{1}{2}} \neq 0 .
$$

Suppose that there exists a point $q \in \partial G$ with $\delta(p)<\delta(q)$. Then, (1.12) gives (2) of Proposition 2.4. In particular, we choose $r=\delta(p), \xi=\nu(p)$ and $\eta=\nu(q)$ to infer that

$$
t^{-\frac{N+1}{4}} \nu(p) \cdot \int_{B_{\delta(p)}(p)} u(x, t)(x-p) d x=t^{-\frac{N+1}{4}} \nu(q) \cdot \int_{B_{\delta(p)}(q)} u(x, t)(x-q) d x \text { for every } t>0 .
$$

On the other hand, it follows from (2) of Lemma 2.1 that the right-hand side of (3.8) tends to 0 as $t \rightarrow+0$, which contradicts (3.7). Therefore, we conclude that $\delta(q) \leq \delta(p)$ for every $q \in \partial G$. Moreover, (3.2) yields that $\delta(q)=R$ for every $q \in \partial G$ and $R>0$. Thus assertion (1) holds also under the assumption (1.12).

Once we have (1) under the assumption (1.12) of Theorem 1.4, we infer that for every $x \in \partial G$ there exists a unique $z=z(x) \in \partial \Omega$ satisfying

$$
\overline{B_{R}(x)} \cap \partial \Omega=\{z(x)\},
$$

since $\partial G$ is of class $C^{1}$. As in [15, Lemma 2.4, pp. 176-179], we introduce the set $\gamma \subset \partial \Omega$ by

$\gamma=\left\{z \in \partial \Omega: \overline{B_{R}(x(z))} \cap \partial \Omega=\{z\}\right.$ for $x(z)=z-R \nu(z) \in \partial G$ and $\left.\max _{1 \leq j \leq N-1} \kappa_{j}(z)<1 / R\right\}$.

Then Lemma 3.2 implies that $\gamma \neq \emptyset$, and assertion (1) yields that

$$
B_{R}(z) \cap G=\emptyset \text { and } \nu(x(z))=\nu(z) \text { for every } z \in \gamma .
$$


Thus, we infer that the formula (3.7) holds if we set $p=x(z) \in \partial G$ with $z \in \gamma$ and $\nu(p) \cdot(z-p)=R=\delta(p)$, that is, for every $z \in \gamma$

$$
\lim _{t \rightarrow+0} t^{-\frac{N+1}{4}} \nu(x(z)) \cdot \int_{B_{R}(x(z))} u(x, t)(x-x(z)) d x=C(N, \sigma) R\left\{\prod_{j=1}^{N-1}\left(\frac{1}{R}-\kappa_{j}(z)\right)\right\}^{-\frac{1}{2}}>0 .
$$

Hence, combining (2) of Proposition 2.4 with this formula (3.10) yields that there exists a number $c>0$ satisfying

$$
\prod_{j=1}^{N-1}\left(\frac{1}{R}-\kappa_{j}(z)\right)=c \text { for every } z \in \gamma .
$$

Then, since $\partial \Omega$ is of class $C^{2}$, combining (3.9) with (3.11) yields that $\gamma$ is closed in $\partial \Omega$. On the other hand, the inverse mapping theorem implies that $\gamma$ is also open in $\partial \Omega$ and the mapping $\gamma \ni z \mapsto x(z) \in \partial G$ is a local diffeomorphism. Therefore $\gamma=\partial \Omega$, since $\partial \Omega$ is connected. Thus the others (3)-(5) follow immediately. Finally, the analyticity of $\partial \Omega$ follows from (5) and hence $\partial G$ is also real analytic by (3). The proof of Lemma 3.1 is completed.

Completion of the proofs of Theorems 1.2 and 1.4: As mentioned in the beginning of this section, Lemma 3.1 implies that $\partial \Omega$ must be a hyperplane under each of the assumptions of Theorems 1.2 and 1.4. Then, by Lemma 3.1, $\partial G$ must be a hyperplane parallel to $\partial \Omega$. Let us prove Theorems 1.2 and 1.4 by using Theorem 5.1 given in section [5.

Let $u$ be the solution of problem (1.3). We introduce the function $w=w(x)\left(x \in \overline{\mathbb{R}^{N}}\right)$ by

$$
w(x)=\int_{0}^{\infty} e^{-t} u(x, t) d t
$$

Then $w$ satisfies

$$
\begin{aligned}
& -\operatorname{div}(\sigma \nabla w)+w=0 \text { in } \Omega, \\
& -\sigma_{m} \Delta(1-w)+(1-w)=0 \text { in } \mathbb{R}^{N} \backslash \bar{\Omega}, \\
& \left.w\right|_{-}=\left.w\right|_{+} \text {and }\left.\sigma_{s} \frac{\partial w}{\partial \nu}\right|_{-}=\left.\sigma_{m} \frac{\partial w}{\partial \nu}\right|_{+} \text {on } \partial \Omega, \\
& 0<w<1 \text { in } \mathbb{R}^{N}, \\
& \lim _{x \notin \Omega, \delta(x) \rightarrow \infty}(1-w(x))=0,
\end{aligned}
$$

where + denotes the limit from outside and - that from inside of $\Omega$ and (3.17) comes from (3) of Lemma 2.1 and Lebesgue's dominated convergence theorem. Then (3.13) and (3.16) 
give (5.1) and (5.2) in section 5, respectively. Thus it suffices to show (5.3). Let $\Theta \in \mathbb{R}^{N}$ be an arbitrary vector parallel to the hyperplanes $\partial \Omega$ and $\partial G$. Consider the function

$$
v^{*}(x, t)=u(x, t)-u(x+\Theta, t) \text { for }(x, t) \in\left(\mathbb{R}^{N} \backslash G\right) \times(0,+\infty) .
$$

Then $v^{*}=v^{*}(x, t)$ satisfies

$$
\begin{array}{ll}
v_{t}^{*}=\operatorname{div}\left(\sigma \nabla v^{*}\right) & \text { in }\left(\mathbb{R}^{N} \backslash \bar{G}\right) \times(0,+\infty), \\
\text { Either } v^{*}=0 & \text { on } \partial G \times(0,+\infty) \text { or } \frac{\partial v^{*}}{\partial \nu}=0 \text { on } \partial G \times(0,+\infty), \\
v^{*}=0 & \text { on }\left(\mathbb{R}^{N} \backslash \bar{G}\right) \times\{0\} .
\end{array}
$$

Hence it follows from the maximum principle that $v^{*} \equiv 0$, that is, in $\left(\mathbb{R}^{N} \backslash G\right) \times(0,+\infty)$, the solution $u$ depends only on $\delta(x)$ and $t$ since $\Theta \in \mathbb{R}^{N}$ is an arbitrary vector parallel to the hyperplane $\partial \Omega$. Therefore $w$ depends only on $\delta(x)$ in $\mathbb{R}^{N} \backslash G$ and hence (5.3) holds true. (3.16) gives the fact that $0<\alpha<1$ in (5.3), and (3.14), (3.15) and (3.17) yield that $\beta>0$. Indeed, by solving (3.14), we get

$$
1-w(x)=c_{0} \exp \left(-\frac{\delta(x)}{\sqrt{\sigma_{m}}}\right) \text { for every } x \in \mathbb{R}^{N} \backslash \Omega
$$

for some positive number $0<c_{0}<1$. This together with (3.15) yields that $\beta>0$. Therefore Theorem 5.1 implies the conclusion of Theorems 1.2 and 1.4 for problem (1.3).

It remains to take care of the solution $u$ of problem (1.4)-(1.6). We introduce the function $w=w(x)(x \in \bar{\Omega})$ by (3.12). Then $w$ satisfies

$$
\begin{aligned}
& -\operatorname{div}(\sigma \nabla w)+w=0 \text { in } \Omega, \\
& 0<w<1 \text { in } \Omega, \\
& w=1 \text { on } \partial \Omega .
\end{aligned}
$$

Hence (3.18) and (3.19) give (5.1) and (5.2) in section 5, respectively. Thus it suffices to show (5.3). Let $\Theta \in \mathbb{R}^{N}$ be an arbitrary vector parallel to the hyperplanes $\partial \Omega$ and $\partial G$. Consider the function

$$
v^{*}(x, t)=u(x, t)-u(x+\Theta, t) \text { for }(x, t) \in(\bar{\Omega} \backslash G) \times(0,+\infty) .
$$

Then $v^{*}=v^{*}(x, t)$ satisfies

$$
\begin{aligned}
& v_{t}^{*}=\operatorname{div}\left(\sigma \nabla v^{*}\right) \quad \text { in }(\Omega \backslash \bar{G}) \times(0,+\infty), \\
& \text { Either } v^{*}=0 \text { on } \partial G \times(0,+\infty) \text { or } \frac{\partial v^{*}}{\partial \nu}=0 \text { on } \partial G \times(0,+\infty), \\
& v^{*}=0 \text { on }[\partial \Omega \times(0,+\infty)] \cup[(\Omega \backslash \bar{G}) \times\{0\}]
\end{aligned}
$$


Hence it follows from the maximum principle that $v^{*} \equiv 0$, that is, in $(\Omega \backslash G) \times(0,+\infty)$, the solution $u$ depends only on $\delta(x)$ and $t$ since $\Theta \in \mathbb{R}^{N}$ is an arbitrary vector parallel to the hyperplane $\partial \Omega$. Therefore $w$ depends only on $\delta(x)$ in $\Omega \backslash G$ and hence (5.3) holds true. (3.20) gives that $\alpha=1$, and it follows from (3.19), (3.20) and Hopf's boundary point lemma that $\beta>0$. Therefore Theorem 5.1 implies the conclusion of Theorems 1.2 and 1.4 for problem (1.4)-(1.6) .

\section{Proofs of Theorems 1.3 and 1.1: the 2nd strategy}

Under the assumptions of Theorems 1.3 and 1.1, we follow the proofs of [4, Theorems 1.4 and 1.5 in section 5] in order to prove that the mean curvature of $\partial \Omega$ is constant. Once this is proved, we immediately infer that $\partial \Omega$ must be a hyperplane. Indeed, since $\partial \Omega$ is an entire graph over $\mathbb{R}^{N-1}$, the constant mean curvature must be zero and if $N=2$ then $\partial \Omega$ must be a straight line, if $3 \leq N \leq 8$, by the Bernstein theorem for the minimal surface equation (see [7, Theorem 17.8, p. 208]), $\partial \Omega$ must be a hyperplane, and if $\nabla f$ is bounded in $\mathbb{R}^{N-1}$ with $N \geq 3$, by Moser's theorem [11, Corollary, p. 591] (see also [7, Theorem 17.5 , p. 205]), the same conclusion holds true.

Since $\partial \Omega$ is uniformly of class $C^{6}$, there exists two positive numbers $r$ and $K$ such that, for every point $p \in \partial \Omega$, there exist an orthogonal coordinate system $z$ and a function $\varphi \in C^{6}\left(\mathbb{R}^{N-1}\right)$ such that the $z_{N}$ coordinate axis lies in the inward normal direction to $\partial \Omega$ at $p$, the origin is located at $p, C^{6}$ norm of $\varphi$ in $\mathbb{R}^{N-1}$ is less than $K, \varphi(0)=0, \nabla \varphi(0)=0$ and the set $B_{r}(p) \cap \Omega$ is written as in the $z$ coordinate system

$$
\left\{z \in B_{r}(0): z_{N}>\varphi\left(z_{1}, \ldots, z_{N-1}\right)\right\}
$$

Since $\partial \Omega$ is uniformly of class $C^{6}$ as explained above, by choosing a number $\delta_{0}>0$ sufficiently small and setting

$$
\mathcal{N}_{-}=\left\{x \in \Omega: 0<\delta(x)<\delta_{0}\right\} \text { and } \mathcal{N}_{+}=\left\{x \in \mathbb{R}^{N} \backslash \bar{\Omega}: 0<\delta(x)<\delta_{0}\right\},
$$

where $\delta(x)$ is the distance function given by (2.1), we see that

$$
\delta \in C^{6}\left(\overline{\mathcal{N}_{ \pm}}\right), \sup \left\{\left|\frac{\partial^{\alpha} \delta}{\partial x^{\alpha}}(x)\right|: x \in \overline{\mathcal{N}_{ \pm}},|\alpha| \leq 6\right\}<+\infty, \sigma= \begin{cases}\sigma_{s} & \text { in } \mathcal{N}_{-} \\ \sigma_{m} & \text { in } \mathcal{N}_{+}\end{cases}
$$

for every $x \in \overline{\mathcal{N}_{ \pm}}$there exists a unique $z=z(x) \in \partial \Omega$ with $\delta(x)=|x-z|$,

$z(x)=x-\delta(x) \nabla \delta(x)$ for all $x \in \overline{\mathcal{N}_{ \pm}}$,

$\max _{1 \leq j \leq N-1}\left|\kappa_{j}(z)\right|<\frac{1}{2 \delta_{0}}$ for every $z \in \partial \Omega$, 
where $\kappa_{1}(z), \ldots, \kappa_{N-1}(z)$ denote the principal curvatures of $\partial \Omega$ at a point $z \in \partial \Omega$ with respect to the inward normal direction $-\nu(z)=\nabla \delta(z)$ to $\partial \Omega$ for $\delta \in C^{6}\left(\overline{\mathcal{N}_{-}}\right)$.

As in the proofs of [4, Theorems 1.4 and 1.5 in section 5], we introduce the function $w=w(x, \lambda)$ by

$$
w(x, \lambda)=\lambda \int_{0}^{\infty} e^{-\lambda t} u(x, t) d t \begin{cases}\text { for }(x, \lambda) \in \bar{\Omega} \times(0,+\infty) & \text { in problem (1.4)-(1.6) } \\ \text { for }(x, \lambda) \in \mathbb{R}^{N} \times(0,+\infty) & \text { in problem (1.3) }\end{cases}
$$

Although the difference between [4, Theorems 1.4 and 1.5] and Theorems 1.3 and 1.1 is such that the neighborhoods of $\partial \Omega$ is bounded in [4, Theorems 1.4 and 1.5] and they are unbounded in Theorems 1.3 and 1.1, we have all the ingredients corresponding to those in [4, Theorems 1.4 and 1.5]; the maximum principle (Proposition A.3) enables us to use the comparison arguments on each of unbounded neighborhoods $\mathcal{N}_{ \pm} ;(2)$ of Lemma 2.1 yields that $w(x, \lambda)$ and $1-w(x, \lambda)$ decay exponentially as $\lambda \rightarrow \infty$ on $\partial \mathcal{N}_{-} \backslash \partial \Omega$ and $\partial \mathcal{N}_{+} \backslash \partial \Omega$, respectively; Proposition 2.3 works for problem (1.3) even if $\partial \Omega$ is unbounded; the situation (4.2)-(4.5) coming from the fact that $\partial \Omega$ is uniformly of class $C^{6}$ enables us to construct the same precise barriers for $w$; and moreover, by introducing an increasing sequence of bounded subdomains in each of $\mathcal{N}_{ \pm}$together with an increasing sequence of bounded harmonic functions on each of the subdomains, we can construct a harmonic function $\psi=\psi(x)$, as the limit of the sequence, on each of $\mathcal{N}_{ \pm}$satisfying

$$
\psi=0 \text { on } \partial \Omega, \psi=2 \text { on } \partial \mathcal{N}_{ \pm} \backslash \partial \Omega \text { and } 0<\psi<2 \text { in } \mathcal{N}_{ \pm},
$$

even if $\mathcal{N}_{ \pm}$is unbounded. This harmonic function $\psi$ was needed in constructing the precise barriers in the proofs of [4, Theorems 1.4 and 1.5]. Therefore, the same arguments as in the proofs of [4, Theorems 1.4 and 1.5 in section 5] work and we conclude that the mean curvature of $\partial \Omega$ must be constant. Thus, as mentioned in the beginning of this section, $\partial \Omega$ must be a hyperplane. Hence, as in the proofs of Theorems 1.2 and 1.4 in section 3 , we may infer that $w(x)=w(x, 1)$ satisfies (5.1) (5.3) with $0<\alpha \leq 1$ and $\beta>0$. Therefore Theorem 5.1 implies the conclusion of Theorems 1.3 and 1.1. $\square$

\section{$5 \quad$ An elliptic overdetermined problem}

In this section, we assume that $\partial \Omega$ is a hyperplane, that is, $f$ is an affine function in (1.1). Moreover, let us assume that there exists a function $w=w(x)(x \in \bar{\Omega})$ which satisfies the 
following:

$$
\begin{aligned}
& -\operatorname{div}(\sigma \nabla w)+w=0 \text { in } \Omega \\
& 0<w<1 \text { in } \Omega \\
& w=\alpha \text { and } \sigma_{s} \frac{\partial w}{\partial \nu}=\beta \text { on } \partial \Omega,
\end{aligned}
$$

where $\nu$ denotes the outward unit normal vector to $\partial \Omega, \sigma$ is given by (1.2) and $\alpha, \beta$ are constants with $0<\alpha \leq 1, \beta>0$, respectively. Define two functions $w_{ \pm}$by

$$
w_{+}(x)=w(x) \text { for } x \in \bar{\Omega} \backslash D \text { and } w_{-}(x)=w(x) \text { for } x \in \bar{D} .
$$

Then the transmission condition for $w$ on $\partial D$ is written as

$$
w_{+}=w_{-} \text {and } \sigma_{s} \frac{\partial w_{+}}{\partial \nu}=\sigma_{c} \frac{\partial w_{-}}{\partial \nu} \text { on } \partial D,
$$

where $\nu$ denotes the outward unit normal vector to $\partial D$.

Theorem 5.1 Suppose that the function $h-f$ has a minimum value in $\mathbb{R}^{N-1}$ and either $h-f$ has a maximum value in $\mathbb{R}^{N-1}$ or $h-f$ is unbounded in $\mathbb{R}^{N-1}$. Then $\partial D$ must be a hyperplane parallel to $\partial \Omega$.

Remark 5.2 We basically follow the arguments in [16] to prove this theorem. However, the difference is such that [16] concerns concentric balls and Theorem 5.1 does parallel hyperplanes; the former is compact and the latter is not compact. As mentioned in section 1. Hopf's boundary point lemma and the transmission condition (5.4) on $\partial D$, together with three comparison principles and one maximum principle for elliptic equations with discontinuous conductivities given in section 6, play a key role.

Proof of Theorem 5.1. Since $\partial \Omega$ is a hyperplane, by a translation and a rotation we may assume that in the new coordinate system $z$

$$
\Omega=\left\{z \in \mathbb{R}^{N}: z_{N}>0\right\} .
$$

Then, with the aid of the uniqueness of the solutions of the Cauchy problem for elliptic equations, we see that $w_{+}$must be a function of one variable $\rho=z_{N}$ and $w_{+}=w_{+}(\rho)$ satisfies

$$
-\sigma_{s} w_{+}^{\prime \prime}(\rho)+w_{+}(\rho)=0 \text { in } \Omega \backslash \bar{D}, w_{+}(0)=\alpha \text { and } \sigma_{s} w_{+}^{\prime}(0)=-\beta .
$$

Moreover we extend $w_{+}$as a unique solution of the above Cauchy problem in (5.5) for all $\rho=z_{N}$ with $z \in \mathbb{R}^{N}$ and we have for some constants $c_{1}, c_{2}$

$$
w_{+}(\rho)=c_{1} \exp \left(-\frac{\rho}{\sqrt{\sigma_{s}}}\right)+c_{2} \exp \left(\frac{\rho}{\sqrt{\sigma_{s}}}\right) \text { for all } \rho \in \mathbb{R} \text {. }
$$


Then it follows from (5.5) that

$$
c_{1}+c_{2}=\alpha \in(0,1], \sqrt{\sigma_{s}}\left(c_{1}-c_{2}\right)=\beta>0 \text { and hence } c_{1}>0 .
$$

In view of the assumption, we may deal with the following two cases in the original coordinate system $x$ :
(I) $h-f$ is unbounded in $\mathbb{R}^{N-1}$;
(II) $h-f$ has a maximum value in $\mathbb{R}^{N-1}$.

Let us consider case (I) first. (5.2) yields that $c_{2}=0$ and hence $0<c_{1} \leq 1$ by (5.5). Thus

$$
w_{+}(\rho)=c_{1} \exp \left(-\frac{\rho}{\sqrt{\sigma_{s}}}\right) \text { with } 0<c_{1} \leq 1 \text { for all } \rho \in \mathbb{R} .
$$

Then we notice that

$$
w_{+}^{\prime}(\rho)<0 \text { for all } \rho \in \mathbb{R} \text { and } \lim _{\rho \rightarrow+\infty} w_{+}(\rho)=0 .
$$

Since the function $h-f$ has a minimum value in $\mathbb{R}^{N-1}$ and $f$ is an affine function in the original coordinate system $x$, there exists a point $z^{*} \in \partial D$ in the new coordinate system $z$ satisfying

$$
z_{N}^{*}=\min _{z \in \partial D} z_{N}>0 \text { and }\left\{z_{N} \in \mathbb{R}: z \in \partial D\right\}=\left[z_{N}^{*}, \infty\right) .
$$

Let $v_{*}=v_{*}(\rho)\left(\rho \geq z_{N}^{*}\right)$ be the unique solution of the Cauchy problem:

$$
-\sigma_{c} v_{*}^{\prime \prime}(\rho)+v_{*}(\rho)=0 \text { for } \rho \in \mathbb{R}, v_{*}\left(z_{N}^{*}\right)=w_{+}\left(z_{N}^{*}\right) \text { and } \sigma_{c} v_{*}^{\prime}\left(z_{N}^{*}\right)=\sigma_{s} w_{+}^{\prime}\left(z_{N}^{*}\right) \text {. }
$$

Hence we have for some constants $c_{3}^{*}, c_{4}^{*}$

$$
v_{*}(\rho)=c_{3}^{*} \exp \left(-\frac{\rho}{\sqrt{\sigma_{c}}}\right)+c_{4}^{*} \exp \left(\frac{\rho}{\sqrt{\sigma_{c}}}\right) \text { for } \rho \in \mathbb{R} .
$$

Distinguish two cases:

$$
\text { (I-1) } \sigma_{c}>\sigma_{s} ; \quad(\mathrm{I}-2) \sigma_{c}<\sigma_{s} .
$$

In case (I-1) we have from (5.8) that

$$
\sigma_{s} w_{+}^{\prime}\left(z_{N}^{*}\right)=\sigma_{c} v_{*}^{\prime}\left(z_{N}^{*}\right)<0 .
$$

Hence, with (5.8) in hand, by applying (2)-(ii) of Proposition A.1 to $w_{1}=w_{+}$and $w_{2}=v_{*}$, we have

$$
w_{+}(\rho)<v_{*}(\rho) \text { for every } \rho>z_{N}^{*} \text {, and hence } c_{4}^{*} \geq 0 \text {. }
$$

We also have

$$
w \not \equiv v_{*} \text { and } w \leq v_{*} \text { on } \partial D
$$


Therefore, since $-\sigma_{c} \Delta w+w=-\sigma_{c} \Delta v_{*}+v_{*}=0$ and $0<w<1$ in $D, c_{4}^{*} \geq 0$ and $\min \left\{v_{*}, 1\right\}$ is a bounded supersolution in $D$, it follows from the comparison principle (Proposition A.3) that

$$
v_{*}>w \text { in } D .
$$

Here we applied Proposition $A .3$ to the function $\min \left\{v_{*}, 1\right\}-w$ in $D$. Thus, with the aid of Hopf's boundary point lemma at $z^{*} \in \partial D$, this contradicts the fact that

$$
v_{*}=w \text { and } \frac{\partial v_{*}}{\partial \nu}=\left.\frac{\partial w}{\partial \nu}\right|_{-}\left(=\frac{\partial w_{-}}{\partial \nu}\right) \text { at } z^{*}
$$

where $\nu$ denotes the outward unit normal vector to $\partial D$ and - denotes the limit from inside of $D$. Here we used (5.4).

In case (I-2), we also have (5.10) from (5.8) and the same argument as in case (I-1), together with Proposition A.1, yields that (5.11) is replaced with

$$
v_{*}(\rho)<w_{+}(\rho) \text { for every } \rho>z_{N}^{*} \text {, and hence } c_{4}^{*} \leq 0,
$$

and then the comparison principle (Proposition A.3) gives

$$
v_{*}<w \text { in } D
$$

since $\max \left\{v_{*}, 0\right\}$ is a bounded subsolution in $D$. Thus we get a contradiction with the aid of Hopf's boundary point lemma at $z^{*} \in \partial D$. Therefore, case (I) does not occur.

Let us proceed to case (II). Since the function $h-f$ has a maximum value in $\mathbb{R}^{N-1}$ and $f$ is an affine function in the original coordinate system $x$, there exists a point $z^{\sharp} \in \partial D$ in the new coordinate system $z$ satisfying

$$
z_{N}^{\sharp}=\max _{z \in \partial D} z_{N}>0 \text { and }\left\{z_{N} \in \mathbb{R}: z \in \partial D\right\}=\left[z_{N}^{*}, z_{N}^{\sharp}\right] .
$$

If $z_{N}^{\sharp}=z_{N}^{*}$, then $\partial D$ must be a hyperplane parallel to $\partial \Omega$ and hence the conclusion of Theorem 5.1 holds true. Therefore we distinguish three cases:

$$
\text { (IIa) } c_{2}=0 \text { and } z_{N}^{\sharp}>z_{N}^{*} ; \quad \text { (IIb) } c_{2}<0 \text { and } z_{N}^{\sharp}>z_{N}^{*} ; \quad \text { (IIc) } c_{2}>0 \text { and } z_{N}^{\sharp}>z_{N}^{*} \text {. }
$$

In case (IIa) (5.6) yields (5.8). Then the same arguments as in case (I) work and we get a contradiction, that is, case (IIa) does not occur.

In case (IIb) we notice that (5.8) is replaced with

$$
w_{+}^{\prime}(\rho)<0 \text { for all } \rho \in \mathbb{R} \text { and } \lim _{\rho \rightarrow+\infty} w_{+}(\rho)=-\infty .
$$

Distinguish two cases:

$$
\text { (IIb-1) } \sigma_{c}>\sigma_{s} ; \quad(\mathrm{IIb}-2) \sigma_{c}<\sigma_{s} \text {. }
$$


With (5.17) in hand, in case (IIb-2) by the same arguments as in case (I-2) we notice that $c_{4}^{*}<0$ and hence we obtain (5.15) which gives a contradiction with the aid of Hopf's boundary point lemma at $z^{*} \in \partial D$. In case (IIb-1), if $c_{4}^{*} \geq 0$, then the same arguments as in case (I-1) also work and one can get a contradiction. Thus it suffices to take care of case (IIb-1) with $c_{4}^{*}<0$.

Let us consider case (IIb-1) with $c_{4}^{*}<0$. For every $r \geq z_{N}^{*}$, we introduce the solution $v_{r}=v_{r}(\rho)(\rho \in \mathbb{R})$ of the Cauchy problem:

$$
-\sigma_{c} v_{r}^{\prime \prime}(\rho)+v_{r}(\rho)=0 \text { for } \rho \in \mathbb{R}, v_{r}(r)=w_{+}(r) \text { and } \sigma_{c} v_{r}^{\prime}(r)=\sigma_{s} w_{+}^{\prime}(r) .
$$

Hence we have for some constants $c_{3}(r), c_{4}(r)$

$$
v_{r}(\rho)=c_{3}(r) \exp \left(-\frac{\rho}{\sqrt{\sigma_{c}}}\right)+c_{4}(r) \exp \left(\frac{\rho}{\sqrt{\sigma_{c}}}\right) \text { for } \rho \in \mathbb{R} .
$$

In particular, we have

$$
c_{4}(r)=\frac{\sqrt{\sigma_{c}} w_{+}(r)+\sigma_{s} w_{+}^{\prime}(r)}{2 \sqrt{\sigma_{c}}} \exp \left(-\frac{r}{\sqrt{\sigma_{c}}}\right) .
$$

Note that $c_{3}\left(z_{N}^{*}\right)=c_{3}^{*}, c_{4}\left(z_{N}^{*}\right)=c_{4}^{*}$ and $v_{z_{N}^{*}}=v_{*}$, where $c_{3}^{*}, c_{4}^{*}$ and $v_{*}$ are given in (5.9). Set

$$
c_{3}^{\sharp}=c_{3}\left(z_{N}^{\sharp}\right), c_{4}^{\sharp}=c_{4}\left(z_{N}^{\sharp}\right) \text { and } v_{\sharp}=v_{z_{N}^{\sharp}} .
$$

Distinguish two cases:

$$
\text { (IIb-1-1) } \quad c_{4}^{\sharp} \leq 0 ; \quad\left(\text { IIb-1-2) } \quad c_{4}^{\sharp}>0 .\right.
$$

In case (IIb-1-1), with (5.17) in hand, the same arguments as in (I) also work and (5.11) is replaced with

$$
v_{\sharp}(\rho)<w_{+}(\rho) \text { for every } \rho<z_{N}^{\sharp} \text {. }
$$

Then we also have

$$
w \not \equiv v_{\sharp} \text { and } w \geq v_{\sharp} \text { on } \partial D,
$$

and the comparison principle (Proposition A.3) gives

$$
v_{\sharp}<w \text { in } D,
$$

since $\max \left\{v_{\sharp}, 0\right\}$ is a bounded subsolution in $D$. Thus we get a contradiction with the aid of Hopf's boundary point lemma at $z^{\sharp} \in \partial D$. Therefore, case (IIb-1-1) does not occur.

In case (IIb-1-2), in view of (5.17) and (5.19), we observe that there exists $R>0$ satisfying

$$
z_{N}^{*}<z_{N}^{\sharp}<R, c_{4}^{*}=c_{4}\left(z_{N}^{*}\right)<0, c_{4}^{\sharp}=c_{4}\left(z_{4}^{\sharp}\right)>0 \text { and } c_{4}(R)<0 .
$$


By (5.19), $c_{4}(r)$ is continuous in $r$. Therefore, it follows from the intermediate value theorem that there exist two numbers $r_{1}$ and $r_{2}$ satisfying

$$
z_{N}^{*}<r_{1}<z_{N}^{\sharp}<r_{2}<R \text { and } c_{4}\left(r_{1}\right)=c_{4}\left(r_{2}\right)=0,
$$

and hence in particular both the functions $v_{r_{j}}(j=1,2)$ are bounded in $[0, \infty)$. Introduce two functions $w_{j}=w_{j}(\rho)(j=1,2)$ for $\rho \geq 0$ by

$$
w_{j}(\rho)= \begin{cases}w_{+}(\rho) & \text { if } 0 \leq \rho \leq r_{j} \\ v_{r_{j}}(\rho) & \text { if } \rho>r_{j}\end{cases}
$$

Then we can apply Proposition A.2 to these $w_{j}=w_{j}(\rho)(j=1,2)$ and obtain that $r_{1}=r_{2}$, which is a contradiction. Therefore, case (IIb-1-2) does not occur.

In case (IIc) it follows that there exists a unique $r_{0}>0$ satisfying

$$
w_{+}^{\prime}(\rho)<0 \text { if } \rho<r_{0}, w_{+}^{\prime}(\rho)>0 \text { if } \rho>r_{0} \text { and } \lim _{\rho \rightarrow+\infty} w_{+}(\rho)=+\infty .
$$

Distinguish three cases:

$$
\text { (IIc-1) } 0<r_{0} \leq z_{N}^{*} ; \quad\left(\text { IIc-2) } \quad z_{N}^{*}<r_{0}<z_{N}^{\sharp} ; \quad\left(\text { IIc-3) } z_{N}^{\sharp} \leq r_{0} .\right.\right.
$$

Let us first consider case (IIc-1). Distinguish two cases:

$$
\text { (IIc-1-1) } \sigma_{c}>\sigma_{s} ; \quad\left(\text { IIc-1-2) } \sigma_{c}<\sigma_{s}\right.
$$

In case (IIc-1-1), we employ $v_{\sharp}$. It follows from (1) of Proposition A.1 that

$$
w_{+}(\rho)<v_{\sharp}(\rho) \text { if } r_{0} \leq \rho<z_{N}^{\sharp} .
$$

Moreover, by integrating the ordinary differential equations which $w_{+}$and $v_{\sharp}$ satisfy, we have

$$
-\sigma_{c} v_{\sharp}^{\prime}\left(r_{0}\right)=-\left(\sigma_{c} v_{\sharp}^{\prime}\left(r_{0}\right)-\sigma_{s} w_{+}^{\prime}\left(r_{0}\right)\right)=\int_{r_{0}}^{z_{n}^{\sharp}}\left(v_{\sharp}(\rho)-w_{+}(\rho)\right) d \rho>0 .
$$

Hence we notice that

$$
v_{\sharp}^{\prime}\left(r_{0}\right)<0 \text { and } v_{\sharp}^{\prime}\left(z_{N}^{\sharp}\right)=\frac{\sigma_{s}}{\sigma_{c}} w_{+}^{\prime}\left(z_{N}^{\sharp}\right)>0 .
$$

This implies that $v_{\sharp}$ must have a critical point and hence $c_{4}^{\sharp}>0$. We also have from (5.24) that

$$
w \not \equiv v_{\sharp} \text { and } w \leq v_{\sharp} \text { on } \partial D \text {. }
$$

Thus the comparison principle (Proposition A.3) gives

$$
v_{\sharp}>w \text { in } D,
$$


since $\min \left\{v_{\sharp}, 1\right\}$ is a bounded supersolution in $D$ because of the fact that $c_{4}^{\sharp}>0$. Thus we get a contradiction with the aid of Hopf's boundary point lemma at $z^{\sharp} \in \partial D$. Therefore, case (IIc-1-1) does not occur.

In case (IIc-1-2), we employ $v_{*}$ instead of $v_{\sharp}$. It follows from (1) of Proposition A.1 that

$$
w_{+}(\rho)<v_{*}(\rho) \text { if } \rho>z_{N}^{*} \text {, and hence } c_{4}^{*}>0 .
$$

Here positivity of $c_{4}^{*}$ comes from that of $c_{2}$. Thus the same comparison arguments yield a contradiction with the aid of Hopf's boundary point lemma at $z^{*} \in \partial D$, and hence case (IIc-1-2) does not occur. Eventually, case (IIc-1) does not occur. We easily know that the same manner as in case (IIc-1) works also in case (IIc-3).

Let us proceed to the remaining case (IIc-2). Here we need Proposition A.5. Distinguish two cases:

$$
\text { (IIc-2-1) } \sigma_{c}>\sigma_{s} ; \quad\left(\text { IIc-2-2) } \sigma_{c}<\sigma_{s}\right.
$$

In case (IIc-2-2), we employ $v_{r_{0}}$. It follows from (3) of Proposition A.1 that

$$
v_{r_{0}}(\rho)>w_{+}(\rho) \text { for every } \rho \neq r_{0} \text {, and hence } c_{4}\left(r_{0}\right)>0 \text {. }
$$

Because of (5.16) there exists a point $z^{0} \in \partial D$ with $z_{N}^{0}=r_{0}$ and moreover

$$
v_{r_{0}}=w \text { and } \nabla v_{r_{0}}=\nabla w=0 \text { at the point } z^{0} \in \partial D .
$$

Then the same comparison arguments yield a contradiction with the aid of Hopf's boundary point lemma at $z^{0} \in \partial D$. Thus, case (IIc-2-2) does not occur.

In case (IIc-2-1), we employ $v_{*}$. It follows from (2) of Proposition A.1 that

$$
w_{+}(\rho)<v_{*}(\rho) \text { if } z_{N}^{*}<\rho \leq r_{0} .
$$

Remark that this inequality is not sufficient for the previous comparison arguments, because of (5.16). For the sake of this reason, by integrating the ordinary differential equations which $w_{+}$and $v_{*}$ satisfy, we have from (5.28)

$$
\sigma_{c} v_{*}^{\prime}\left(r_{0}\right)=\sigma_{c} v_{*}^{\prime}\left(r_{0}\right)-\sigma_{s} w_{+}^{\prime}\left(r_{0}\right)=\int_{z_{N}^{*}}^{r_{0}}\left(v_{*}(\rho)-w_{+}(\rho)\right) d \rho>0 .
$$

Hence $v_{*}^{\prime}\left(r_{0}\right)>0$. By choosing a constant $\gamma>0$ satisfying

$$
v_{*}\left(r_{0}\right)=\gamma \exp \left(-\frac{r_{0}}{\sqrt{\sigma_{c}}}\right)
$$


we introduce a function $v_{* *}=v_{* *}(\rho)$ for $\rho \geq 0$ given by

$$
v_{* *}(\rho)= \begin{cases}\gamma \exp \left(-\frac{\rho}{\sqrt{\sigma_{c}}}\right) & \text { if } r_{0} \leq \rho, \\ v_{*}(\rho) & \text { if } z_{N}^{*} \leq \rho<r_{0} \\ w_{+}(\rho) & \text { if } 0 \leq \rho<z_{N}^{*} .\end{cases}
$$

Hence we have in particular

$$
\left(\sigma_{c} v_{* *}^{\prime}(\rho)-\sigma_{s} w_{+}^{\prime}(\rho)\right)\left(v_{* *}^{\prime}(\rho)-w_{+}^{\prime}(\rho)\right)>0 \text { if } z_{N^{*}}<\rho<z_{N}^{\sharp} .
$$

Indeed, for $z_{N} *<\rho \leq r_{0}$, by integrating the ordinary differential equations which $w_{+}$and $v_{*}$ satisfy, we have from (5.28)

$$
\sigma_{c} v_{* *}^{\prime}(\rho)-\sigma_{s} w_{+}^{\prime}(\rho)=\sigma_{c} v_{*}^{\prime}(\rho)-\sigma_{s} w_{+}^{\prime}(\rho)=\int_{z_{N}^{*}}^{\rho}\left(v_{*}(s)-w_{+}(s)\right) d s>0 .
$$

Then, since $w_{+}^{\prime}(\rho)<0$ and $\sigma_{c}>\sigma_{s}$, we have

$$
v_{* *}^{\prime}(\rho)-w_{+}^{\prime}(\rho)=\frac{1}{\sigma_{c}}\left(\sigma_{c} v_{* *}^{\prime}(\rho)-\sigma_{c} w_{+}^{\prime}(\rho)\right)>\frac{1}{\sigma_{c}}\left(\sigma_{c} v_{* *}^{\prime}(\rho)-\sigma_{s} w_{+}^{\prime}(\rho)\right)>0 .
$$

Therefore, for $z_{N^{*}}<\rho \leq r_{0}$, inequality (5.29) holds true. For $r_{0}<\rho<z_{N}^{\sharp}$, since $v_{* *}^{\prime}(\rho)<0$ and $w_{+}^{\prime}(\rho)>0$, inequality (5.29) follows easily. Moreover, since $v_{* *}^{\prime}\left(r_{0}-0\right)>$ $0>v_{* *}^{\prime}\left(r_{0}+0\right)$ and $v_{* *}\left(r_{0}-0\right)=v_{* *}\left(r_{0}+0\right)$, we see that

$$
-\left(\sigma_{2} v_{* *}^{\prime}\right)^{\prime}+v_{* *} \geq 0 \text { in }(0, \infty)
$$

where we set

$$
\sigma_{2}=\sigma_{2}(\rho)= \begin{cases}\sigma_{s} & \text { if } 0 \leq \rho \leq z_{N}^{*} \\ \sigma_{c} & \text { if } \rho>z_{N}^{*} .\end{cases}
$$

Then we can apply Proposition A.5 to $w_{1}=w, w_{2}=v_{* *}, \ell=z_{N}^{*}$ and $L=z_{N}^{\sharp}$ and conclude that

$$
w \leq v_{* *} \text { in } \Omega \text {, and hence } w<v_{* *} \text { in } D .
$$

Therefore, this yields a contradiction with the aid of Hopf's boundary point lemma at $z^{*} \in \partial D$, and case (IIc-2-1) does not occur. The proof of Theorem 5.1 is complete.

\section{Appendices}

We deal with three comparison principles and one maximum principle for elliptic equations with discontinuous conductivities. We start with a comparison principle for two solutions of ordinary differential equations with different conductivities (see Lemma 3.5 in [16]). 
Proposition A.1 Let $\sigma_{j}(j=1,2)$ be two constants with $0<\sigma_{1}<\sigma_{2}$ and let $w_{j}=$ $w_{j}(\rho)(j=1,2)$ solve $-\sigma_{j} w_{j}^{\prime \prime}+w_{j}=0$ in $\mathbb{R}$ for $j=1,2$, respectively. Suppose that $w_{1}(r)=w_{2}(r)$ for some $r \in \mathbb{R}$. Then the following assertions hold:

(1) Assume that $\sigma_{1} w_{1}^{\prime}(r)=\sigma_{2} w_{2}^{\prime}(r)>0$. Then we have

(i) If there exists $s \in(-\infty, r)$ such that $w_{1}(s)=w_{2}(s)$ and $w_{1}(\rho)<w_{2}(\rho)$ for every $\rho \in(s, r)$, then $w_{1}^{\prime}(s)<0$ and $w_{2}^{\prime}(s)<0$.

(ii) If there exists $\ell \in(r, \infty)$ such that $w_{1}(\ell)=w_{2}(\ell)$ and $w_{1}(\rho)>w_{2}(\rho)$ for every $\rho \in(r, \ell)$, then $w_{1}^{\prime}(\ell)<0$ and $w_{2}^{\prime}(\ell)<0$.

(2) Assume that $\sigma_{1} w_{1}^{\prime}(r)=\sigma_{2} w_{2}^{\prime}(r)<0$. Then we have

(i) If there exists $s \in(-\infty, r)$ such that $w_{1}(s)=w_{2}(s)$ and $w_{1}(\rho)>w_{2}(\rho)$ for every $\rho \in(s, r)$, then $w_{1}^{\prime}(s)>0$ and $w_{2}^{\prime}(s)>0$.

(ii) If there exists $\ell \in(r, \infty)$ such that $w_{1}(\ell)=w_{2}(\ell)$ and $w_{1}(\rho)<w_{2}(\rho)$ for every $\rho \in(r, \ell)$, then $w_{1}^{\prime}(\ell)>0$ and $w_{2}^{\prime}(\ell)>0$.

(3) If $w_{1}^{\prime}(r)=w_{2}^{\prime}(r)=0$ and $w_{1}(r)=w_{2}(r)>0$, then $w_{1}(\rho)>w_{2}(\rho)$ for every $\rho \neq r$.

Proof. Let us first consider (3). Set $w_{1}(r)=w_{2}(r)=a>0$. Then it follows that for $j=1,2$,

$$
w_{j}(\rho)=\frac{a}{2}\left\{\exp \left(-\frac{\rho-r}{\sqrt{\sigma_{j}}}\right)+\exp \left(\frac{\rho-r}{\sqrt{\sigma_{j}}}\right)\right\} \text { for every } \rho \in \mathbb{R} .
$$

Since $0<\sigma_{1}<\sigma_{2}$, we have the conclusion.

Let us proceed to (1). Note that

$$
\sigma_{1} w_{1}^{\prime \prime}(\rho)-\sigma_{2} w_{2}^{\prime \prime}(\rho)=w_{1}(\rho)-w_{2}(\rho) \text { for } \rho \in \mathbb{R}
$$

Since $\sigma_{1} w_{1}^{\prime}(r)=\sigma_{2} w_{2}^{\prime}(r)>0, w_{1}(r)=w_{2}(r)$ and $0<\sigma_{1}<\sigma_{2}$, we observe that

$$
w_{1}^{\prime}(r)>w_{2}^{\prime}(r),
$$

and hence there exists a number $\delta>0$ such that

$$
w_{1}(\rho)<w_{2}(\rho) \text { for every } \rho \in(r-\delta, r) \text { and } w_{1}(\rho)>w_{2}(\rho) \text { for every } \rho \in(r, r+\delta) \text {. }
$$

Let us prove (i). Since $\sigma_{1} w_{1}^{\prime}(r)=\sigma_{2} w_{2}^{\prime}(r), w_{1}(s)=w_{2}(s)$ and $w_{1}(\rho)<w_{2}(\rho)$ for every $\rho \in(s, r)$, we notice that $w_{1}^{\prime}(s) \leq w_{2}^{\prime}(s)$. Integrating (A.1) over the interval $[s, r]$ gives

$$
-\sigma_{1} w_{1}^{\prime}(s)+\sigma_{2} w_{2}^{\prime}(s)=\int_{s}^{r}\left(w_{1}(\rho)-w_{2}(\rho)\right) d \rho<0 .
$$


These yield that $w_{1}^{\prime}(s)<0$ and $w_{2}^{\prime}(s)<0$, since $0<\sigma_{1}<\sigma_{2}$. (ii) is proved similarly.

It remains to consider $(2)$. Since $\sigma_{1} w_{1}^{\prime}(r)=\sigma_{2} w_{2}^{\prime}(r)<0, w_{1}(r)=w_{2}(r)$ and $0<\sigma_{1}<$ $\sigma_{2}$, we observe that

$$
w_{1}^{\prime}(r)<w_{2}^{\prime}(r),
$$

and hence there exists a number $\delta>0$ such that

$$
w_{1}(\rho)>w_{2}(\rho) \text { for every } \rho \in(r-\delta, r) \text { and } w_{1}(\rho)<w_{2}(\rho) \text { for every } \rho \in(r, r+\delta) \text {. }
$$

Thus the conclusion follows from the same argument as in (1).

We have a proposition concerning the unique determination of discontinuity of the conductivity for an ordinary differential equation with a nontrivial Cauchy data (see Lemma 3.1 in [16] for the case dealing with bounded domains).

Proposition A.2 Let $0<r_{1} \leq r_{2}<\infty$. Define $\sigma_{j}=\sigma_{j}(\rho)(j=1,2)$ for $\rho \geq 0$ by

$$
\sigma_{j}(\rho)= \begin{cases}\sigma_{s} & \text { if } 0 \leq \rho \leq r_{j}, \\ \sigma_{c} & \text { if } r_{j}<\rho,\end{cases}
$$

where $\sigma_{c}, \sigma_{s}$ are positive constants with $\sigma_{c} \neq \sigma_{s}$. Let $w_{j}=w_{j}(\rho)(j=1,2)$ be bounded solutions of $-\left(\sigma_{j} w_{j}^{\prime}\right)^{\prime}+w_{j}=0$ in $[0, \infty)$ satisfying

$$
\begin{aligned}
& w_{1}(0)=w_{2}(0), \quad w_{1}^{\prime}(0)=w_{2}^{\prime}(0), \\
& \text { and either } w_{1}(0) \neq 0 \text { or } w_{1}^{\prime}(0) \neq 0 .
\end{aligned}
$$

Then $r_{1}=r_{2}$ and $w_{1} \equiv w_{2}$ in $[0, \infty)$.

Proof. Since $w_{j}(j=1,2)$ are bounded, we see that there exist two constants $c_{j}(j=1,2)$ satisfying

$$
w_{j}(\rho)=c_{j} \exp \left(-\frac{\rho}{\sqrt{\sigma_{c}}}\right) \text { for every } \rho \geq \rho_{j} \text { and for } j=1,2 .
$$

Transmission conditions yield that $w_{j}(j=1,2)$ are continuous on $[0, \infty)$ and

$$
\sigma_{s} w_{j}^{\prime}\left(r_{j}-0\right)=\sigma_{c} w_{j}^{\prime}\left(r_{j}+0\right) \text { for } j=1,2 \text {. }
$$

Hence we have

$$
\begin{aligned}
& \int_{0}^{\infty} w_{1} w_{2} d x=\int_{0}^{r_{1}}\left(\sigma_{s} w_{1}^{\prime}\right)^{\prime} w_{2} d x+\int_{r_{1}}^{\infty}\left(\sigma_{c} w_{1}^{\prime}\right)^{\prime} w_{2} d x \\
& =-\sigma_{s} w_{1}^{\prime}(0) w_{2}(0)+\sigma_{s} w_{1}^{\prime}\left(r_{1}-0\right) w_{2}\left(r_{1}\right)-\sigma_{c} w_{1}^{\prime}\left(r_{1}+0\right) w_{2}\left(r_{1}\right)-\int_{0}^{\infty} \sigma_{1} w_{1}^{\prime} w_{2}^{\prime} d x \\
& =-\sigma_{s} w_{1}^{\prime}(0) w_{2}(0)-\int_{0}^{\infty} \sigma_{1} w_{1}^{\prime} w_{2}^{\prime} d x
\end{aligned}
$$


Thus we obtain

$$
\int_{0}^{\infty} w_{1} w_{2} d x=-\sigma_{s} w_{1}^{\prime}(0) w_{2}(0)-\int_{0}^{\infty} \sigma_{1} w_{1}^{\prime} w_{2}^{\prime} d x
$$

Changing the roles of $w_{j}(j=1,2)$ yields that

$$
\int_{0}^{\infty} w_{1} w_{2} d x=-\sigma_{s} w_{2}^{\prime}(0) w_{1}(0)-\int_{0}^{\infty} \sigma_{2} w_{1}^{\prime} w_{2}^{\prime} d x
$$

In the same way we also have

$$
\begin{aligned}
& \int_{0}^{\infty} w_{1}^{2} d x=-\sigma_{s} w_{1}^{\prime}(0) w_{1}(0)-\int_{0}^{\infty} \sigma_{1}\left(w_{1}^{\prime}\right)^{2} d x \\
& \int_{0}^{\infty} w_{2}^{2} d x=-\sigma_{s} w_{2}^{\prime}(0) w_{2}(0)-\int_{0}^{\infty} \sigma_{2}\left(w_{2}^{\prime}\right)^{2} d x
\end{aligned}
$$

Therefore by combing (A.2) and (A.3) with the initial condition we obtain

$$
\int_{r_{1}}^{r_{2}} w_{1}^{\prime} w_{2}^{\prime} d x=0
$$

since $\sigma_{s} \neq \sigma_{c}$. Then it follows from these equalities and the initial condition that

$$
\begin{aligned}
& \int_{0}^{\infty}\left(w_{1}-w_{2}\right)^{2} d x \\
& =-\int_{r_{1}}^{r_{2}}\left(\sigma_{c}\left(w_{1}^{\prime}\right)^{2}+\sigma_{s}\left(w_{2}^{\prime}\right)^{2}\right) d x-\int_{0}^{r_{1}} \sigma_{s}\left(w_{1}^{\prime}-w_{2}^{\prime}\right)^{2} d x-\int_{r_{2}}^{\infty} \sigma_{c}\left(w_{1}^{\prime}-w_{2}^{\prime}\right)^{2} d x \\
& \leq 0
\end{aligned}
$$

which yields that $w_{1} \equiv w_{2}$. Moreover, since $w_{1}$ is not constant because of the initial condition, it follows that $r_{1}=r_{2}$.

Let us next give a maximum principle for an elliptic equation in unbounded domains in $\mathbb{R}^{N}$, whose proof can be modified in proving the next key proposition.

Proposition A.3 Let $D \subset \mathbb{R}^{N}$ be an unbounded domain, and let $\sigma=\sigma(x)(x \in D)$ be a general conductivity satisfying

$$
0<\mu \leq \sigma(x) \leq M \quad \text { for every } x \in \mathbb{R}^{N},
$$

where $\mu, M$ are positive constants. Assume that $w \in H_{\text {loc }}^{1}(D) \cap L^{\infty}(D) \cap C^{0}(\bar{D})$ satisfies

$$
-\operatorname{div}(\sigma \nabla w)+\lambda w \geq 0 \text { in } D \text { and } w \geq 0 \text { on } \partial D
$$

for some constant $\lambda>0$. Then $w \geq 0$ in $D$, and moreover, either $w>0$ in $D$ or $w \equiv 0$ in $D$. 
Remark A.4 When $D$ is bounded, this proposition is well known and holds true for every $\lambda \geq 0$. However, when $D$ is unbounded, this proposition is not true for $\lambda=0$. Indeed, a counterexample is given in [3, p. 37], where $N \geq 3, D=\left\{x \in \mathbb{R}^{N}:|x|>1\right\}, \sigma(x) \equiv 1$ and $w(x)=|x|^{2-N}-1$.

Proof of Proposition A.3. Define $v=v(x)$ by

$$
v(x)=e^{-\delta|x|} w(x) \text { for } x \in \bar{D},
$$

where $\delta>0$ is a constant which will be chosen later. Then $v \in H_{l o c}^{1}(D) \cap L^{\infty}(D) \cap C^{0}(\bar{D})$ and moreover

$$
\lim _{|x| \rightarrow \infty} v(x)=0,
$$

since $w \in L^{\infty}(D)$. For every $\varepsilon>0$, we consider a nonnegative function

$$
\varphi(x)=\max \{-\varepsilon-v(x), 0\} \text { for } x \in \bar{D} .
$$

Since $v \in H_{l o c}^{1}(D) \cap L^{\infty}(D) \cap C^{0}(\bar{D})$ and $v \geq 0$ on $\partial D$, it follows from (A.7) that $\varphi$ is compactly supported in $D$ and $\varphi \in H_{0}^{1}(D)$, and hence $e^{-2 \delta|\cdot|} \varphi(\cdot) \in H_{0}^{1}(D)$. Therefore we obtain

$$
\begin{aligned}
0 & \leq \int_{D}\left\{\sigma(x) \nabla w(x) \cdot \nabla\left(\varphi(x) e^{-2 \delta|x|}\right)+\lambda w(x) \varphi(x) e^{-2 \delta|x|}\right\} d x \\
& =\int_{D \cap\{v<-\varepsilon\}} \sigma e^{-\delta|x|}\left\{\left(\delta v \frac{x}{|x|}+\nabla v\right) \cdot\left(\nabla \varphi-2 \delta \varphi \frac{x}{|x|}\right)+\frac{\lambda}{\sigma} v \varphi\right\} d x .
\end{aligned}
$$

Notice that

$$
\varphi(x)=\left\{\begin{array}{ll}
-\varepsilon-v(x) & \text { if } v(x)<-\varepsilon, \\
0 & \text { if } v(x) \geq-\varepsilon,
\end{array} \quad \text { and } \quad \nabla \varphi(x)= \begin{cases}-\nabla v(x) & \text { if } v(x)<-\varepsilon \\
0 & \text { if } v(x) \geq-\varepsilon .\end{cases}\right.
$$

By setting

$$
I=\sigma^{-1} e^{\delta|x|} \times \text { the integrand of the integral (A.8), }
$$

we have

$$
\begin{aligned}
I & =-|\nabla v|^{2}-\frac{\lambda}{\sigma} v^{2}+2 \delta^{2} v^{2}+\delta v \frac{x}{|x|} \cdot \nabla v+\varepsilon\left(2 \delta^{2} v+2 \delta \frac{x}{|x|} \cdot \nabla v-\frac{\lambda}{\sigma} v\right) \\
& \leq-\left\{1-\delta\left(\frac{1}{2}+\varepsilon\right)\right\}|\nabla v|^{2}-\left\{\frac{\lambda}{\sigma}\left(1-\frac{\varepsilon}{2}\right)-\left(2 \delta^{2}+\frac{\delta}{2}\right)\right\} v^{2}+\varepsilon\left(\frac{\lambda}{2 \sigma}+\delta\right) .
\end{aligned}
$$


Here we have used Cauchy's inequality $2 a b \leq a^{2}+b^{2}$ and the fact that $v<0$ in the integrand of (A.8). Therefore, since $0<\mu \leq \sigma(x) \leq M$, we can choose $\delta>0$ sufficiently small to obtain that if $0<\varepsilon<1$ then

$$
I \leq-\frac{1}{4}\left(|\nabla v|^{2}+\frac{\lambda}{M} v^{2}\right)+\varepsilon\left(\frac{\lambda}{2 \mu}+\delta\right)
$$

and hence

$$
\mu \int_{D \cap\{v<-\varepsilon\}} e^{-\delta|x|}\left(|\nabla v|^{2}+\frac{\lambda}{M} v^{2}\right) d x \leq M \varepsilon\left(\frac{2 \lambda}{\mu}+4 \delta\right) \int_{D} e^{-\delta|x|} d x .
$$

By choosing a sequence $\left\{\varepsilon_{n}\right\}$ with $\varepsilon_{n} \downarrow 0$ as $n \rightarrow \infty$ and letting $n \rightarrow \infty$, we conclude that

$$
\int_{D \cap\{v<0\}} e^{-\delta|x|}\left(|\nabla v|^{2}+\frac{\lambda}{M} v^{2}\right) d x=0
$$

and hence $v \geq 0$ in $D$. Therefore $w \geq 0$ in $D$. Once this is shown, the last part follows from the strong maximum principle (see [8, Theorem 8.19, pp. 198-199]).

Finally, we give a comparison principle for two solutions of differential inequalities with different conductivities on a half-space of $\mathbb{R}^{N}$ (see Lemma 3.3 in [16] for the case dealing with bounded domains).

Proposition A.5 For two numbers $L>\ell>0$, set

$$
\Omega=\left\{z \in \mathbb{R}^{N}: z_{N}>0\right\}, E=\left\{z \in \mathbb{R}^{N}: z_{N}>\ell\right\} \quad \text { and } F=\left\{z \in \mathbb{R}^{N}: z_{N}>L\right\} .
$$

Let $D \subset \mathbb{R}^{N}$ be a domain with $C^{2}$ boundary $\partial D$ satisfying that $F \subset D \subset E$. Let $\sigma_{j}=$ $\sigma_{j}(z)(j=1,2)$ be given by

$$
\sigma_{1}=\left\{\begin{array}{ll}
\sigma_{c} & \text { in } D, \\
\sigma_{s} & \text { in } \Omega \backslash D,
\end{array} \quad \sigma_{2}= \begin{cases}\sigma_{c} & \text { in } E, \\
\sigma_{s} & \text { in } \Omega \backslash E,\end{cases}\right.
$$

where $\sigma_{c}, \sigma_{s}$ are positive constants with $\sigma_{c} \neq \sigma_{s}$. Let $w_{1} \in H_{l o c}^{1}(\Omega) \cap L^{\infty}(\Omega), w_{2} \in$ $H_{l o c}^{1}((0, \infty)) \cap L^{\infty}((0, \infty))$ satisfy

$$
\begin{aligned}
& -\operatorname{div}\left(\sigma_{1} \nabla w_{1}\right)+w_{1}=0 \quad \text { in } \quad \Omega, \quad-\left(\sigma_{2} w_{2}^{\prime}\right)^{\prime}+w_{2} \geq 0 \quad \text { in }(0, \infty), \\
& \text { and } w_{1}(z) \equiv w_{2}\left(z_{N}\right) \quad \text { for } z \in \Omega \backslash E .
\end{aligned}
$$

Then, if

$$
\left(\sigma_{c} w_{2}^{\prime}\left(z_{N}\right)-\sigma_{s} \frac{\partial w_{1}}{\partial z_{N}}(z)\right)\left(w_{2}^{\prime}\left(z_{N}\right)-\frac{\partial w_{1}}{\partial z_{N}}(z)\right) \geq 0 \text { for } z \in E \backslash \bar{D}
$$

we have that $w_{1}(z) \leq w_{2}\left(z_{N}\right)$ for $z \in \Omega$. 
Proof. We modify the proof of Proposition A.3. First of all, we extend $w_{2}$ for $z=$ $\left(z_{1}, \ldots, z_{N}\right) \in \Omega$ by $w_{2}(z)=w_{2}\left(z_{N}\right)$. Introduce a function $\psi=\psi(t)(t \in \mathbb{R})$ by

$$
\psi(t)= \begin{cases}e^{-\delta(t-L)} & \text { if } t>L \\ 1 & \text { if } t \leq L\end{cases}
$$

where $\delta>0$ is a constant which will be chosen later. Then we define $v=v(z)$ by

$$
v(z)=e^{-\delta|\hat{z}|} \psi\left(z_{N}\right)\left(w_{2}(z)-w_{1}(z)\right) \text { for } z \in \bar{\Omega},
$$

where $\hat{z}=\left(z_{1}, \ldots, z_{N-1}\right) \in \mathbb{R}^{N-1}$. Note that $v=0$ in $\Omega \backslash E$. If $0<\varepsilon<1$, we set

$$
\varphi(z)=\max \{-\varepsilon-v(z), 0\}(\geq 0) \text { for } z \in \Omega \text {. }
$$

Since $\varphi$ is compactly supported in $\Omega$, we notice that the function $\varphi(z) e^{-2 \delta|\hat{z}|} \psi^{2}\left(z_{N}\right)$ belongs to $H_{0}^{1}(\Omega)$. Therefore we observe that

$$
0 \leq \int_{\Omega}\left\{\left(\sigma_{2}(z) \nabla w_{2}(z)-\sigma_{1}(z) \nabla w_{1}(z)\right) \cdot \nabla\left(\varphi(z) e^{-2 \delta|\hat{z}|} \psi^{2}\left(z_{N}\right)\right)+v(z) \varphi(z) e^{-\delta|\hat{z}|} \psi\left(z_{N}\right)\right\} d z .
$$

Then, since $\varphi=0$ in $\Omega \backslash E$, we have

$$
\begin{aligned}
& 0 \leq \int_{E \backslash D}\left(\sigma_{c} \nabla w_{2}(z)-\sigma_{s} \nabla w_{1}(z)\right) \cdot \nabla\left(\varphi(z) e^{-2 \delta|\hat{z}|} \psi^{2}\left(z_{N}\right)\right) d z \\
& +\int_{D} \sigma_{c} \nabla\left(w_{2}(z)-w_{1}(z)\right) \cdot \nabla\left(\varphi(z) e^{-2 \delta|\hat{z}|} \psi^{2}\left(z_{N}\right)\right) d z+\int_{E} v(z) \varphi(z) e^{-\delta|\hat{z}|} \psi\left(z_{N}\right) d z .
\end{aligned}
$$

By observing that $\psi=1$ and $w_{1}$ depends only on $z_{N}$ in $E \backslash D$, we see that the integral of (A.9) equals

$$
-\int_{(E \backslash D) \cap\{v<-\varepsilon\}}\left(\sigma_{c} \frac{\partial w_{2}}{\partial z_{N}}(z)-\sigma_{s} \frac{\partial w_{1}}{\partial z_{N}}(z)\right)\left(\frac{\partial w_{2}}{\partial z_{N}}(z)-\frac{\partial w_{1}}{\partial z_{N}}(z)\right) e^{-3 \delta|\hat{z}|} d z(\leq 0) .
$$

As for the first integral of (A.10), since we observe that

$$
\left|\nabla\left(e^{\delta|\hat{z}|}\left(\psi\left(z_{N}\right)\right)^{-1}\right)\right| \leq 2 \delta e^{\delta|\hat{z}|}\left(\psi\left(z_{N}\right)\right)^{-1} \text { and }\left|\nabla\left(e^{-2 \delta|\hat{z}|} \psi^{2}\left(z_{N}\right)\right)\right| \leq 4 \delta e^{-2 \delta|\hat{z}|} \psi^{2}\left(z_{N}\right)
$$

the first integral of (A.10) is bounded from above by

$$
\underset{D \cap\{v<-\varepsilon\}}{\sigma_{c}} \int_{D}^{-\delta|\hat{z}|} \psi\left(z_{N}\right)\left\{-|\nabla v|^{2}+4 \delta|\nabla v| \varphi+2 \delta|v||\nabla \varphi|+8 \delta^{2}|v| \varphi\right\} d z .
$$


Moreover, since $\varphi=-\varepsilon-v$, with Cauchy's inequality in hand, we see that the first integral of (A.10) is bounded from above by

$$
\underset{D \cap\{v<-\varepsilon\}}{\sigma_{c}} \int^{-\delta|\hat{z}|} \psi\left(z_{N}\right)\left\{-(1-3 \delta)|\nabla v|^{2}+\delta(3+8 \delta)|v|^{2}\right\} d z .
$$

On the other hand, since $0<\varepsilon<1$, the second integral of (A.10) is bounded from above by

$$
\int_{E \cap\{v<-\varepsilon\}} e^{-\delta|\hat{z}|} \psi\left(z_{N}\right)\left(-\frac{1}{2}|v|^{2}+\frac{1}{2} \varepsilon\right) d z .
$$

Therefore, in view of (A.9) and (A.10), since $D \subset E$, we choose $\delta>0$ sufficiently small to conclude that if $0<\varepsilon<1$ then

$$
\sigma_{c} \int_{D \cap\{v<-\varepsilon\}} e^{-\delta|\hat{z}|} \psi\left(z_{N}\right)|\nabla v|^{2} d z+\int_{E \cap\{v<-\varepsilon\}} e^{-\delta|\hat{z}|} \psi\left(z_{N}\right)|v|^{2} d z \leq 2 \varepsilon \int_{E} e^{-\delta|\hat{z}|} \psi\left(z_{N}\right) d z .
$$

By choosing a sequence $\left\{\varepsilon_{n}\right\}$ with $\varepsilon_{n} \downarrow 0$ as $n \rightarrow \infty$ and letting $n \rightarrow \infty$, we infer that

$$
\sigma_{c} \int_{D \cap\{v<0\}} e^{-\delta|\hat{z}|} \psi\left(z_{N}\right)|\nabla v|^{2} d z+\int_{E \cap\{v<0\}} e^{-\delta|\hat{z}|} \psi\left(z_{N}\right)|v|^{2} d z=0
$$

and hence $v \geq 0$ in $E$, which completes the proof.

\section{References}

[1] G. Alessandrini and N. Garofalo, Symmetry for degenerate parabolic equations, Arch. Rational Mech. Anal., 108 (1989), 161-174.

[2] D. G. Aronson, Bounds for the fundamental solutions of a parabolic equation, Bull. Amer. Math. Soc., 73 (1967), 890-896.

[3] S. Axler, P. Bourdon and W. Ramey, Harmonic Function Theory, (Second Edition), Springer-Verlag New York, 2001.

[4] L. Cavallina, R. Magnanini and S. Sakaguchi, Two-phase heat conductors with a surface of the constant flow property, J. Geom. Anal., (2019), https://doi.org/10.1007/s12220-019-00262-8.

[5] E. Fabes and D. Stroock, A new proof of Moser's parabolic Harnack inequality using the old ideas of Nash, Arch. Rational Mech. Anal., 96 (1986), 327-338. 
[6] N. Garofalo and E. Sartori, Symmetry in a free boundary problem for degenerate parabolic equations on unbounded domains, Proc. Amer. Math. Soc., 129 (2001), 3603-3610.

[7] E. Giusti, Minimal Surfaces and Functions of Bounded Variations, Birkhäuser, Boston, Basel, Stuttgart, 1984.

[8] D. Gilbarg and N. S. Trudinger, Elliptic Partial Differential Equations of Second Order, (Second Edition), Springer-Verlag, Berlin, Heidelberg, New York, Tokyo, 1983.

[9] H. Kang, H. Lee, and S. Sakaguchi, An over-determined boundary value problem arising from neutrally coated inclusions in three dimensions, Ann. Sc. Norm. Sup. Pisa, Cl. Sci., (5) 16 (2016), 1193-1208.

[10] R. Magnanini and S. Sakaguchi, Matzoh ball soup: Heat conductors with a stationary isothermic surface, Ann. of Math., 156 (2002), 931-946.

[11] J. Moser, On Harnack's theorem for elliptic differential equations, Comm. Pure Appl. Math., 14 (1961), 577-591.

[12] M. Ohnuma and S. Sakaguchi, A simple proof of a strong comparison principle for semicontinuous viscosity solutions of the prescribed mean curvature equation, Nonlinear Anal., 181 (2019), 180-188.

[13] A. Savo, Heat flow, heat content and the isoparametric property, Math. Ann., 366 (2016), 1089-1136.

[14] S. Sakaguchi, Stationary level surfaces and Liouville-type theorems characterizing hyperplanes, in " Geometric Properties of Parabolic and Elliptic PDE's ", Springer INdAM Series, Vol. 2, 2013, 269-282.

[15] S. Sakaguchi, Two-phase heat conductors with a stationary isothermic surface, Rendiconti dell'Istituto di Matematica dell'Università di Trieste, 48 (2016), 167-187.

[16] S. Sakaguchi, Two-phase heat conductors with a stationary isothermic surface and their related elliptic overdetermined problems, arXiv:1705.10628v2, RIMS Kôkyûroku Bessatsu, to appear.

[17] J. Serrin, A symmetry problem in potential theory, Arch. Rational Mech. Anal., 43 (1971), 304-318. 